\title{
EXPLORING STAR FORMATION IN HIGH-MASS GALAXIES IN THE LOW-Z UNIVERSE
}

\author{
A Thesis presented to \\ the Faculty of the Graduate School \\ at the University of Missouri
}

In Partial Fulfillment

of the Requirements for the Degree

Doctor of Philosophy

by

James Runge

Dr. Haojing Yan, Thesis Supervisor

May 2018 
The undersigned, appointed by the Dean of the Graduate School, have examined the dissertation entitled:

\section{EXPLORING STAR FORMATION IN HIGH-MASS GALAXIES IN THE LOW-Z UNIVERSE}

presented by James Runge, a candidate for the degree of Doctor of Philosophy and hereby certify that, in their opinion, it is worthy of acceptance.

Dr. Haojing Yan

Dr. Adam Helfer

Dr. Sergei Kopeikin

Dr. Aigen Li

Dr. Angela Speck 
Thank you to my family, my friends, all the cats (too many to name), and to those I've lost along the way 


\section{ACKNOWLEDGMENTS}

I would like to thank my advisor Haojing Yan for his help and guidance with all of my research. I would also like to thank my fellow group members for answering a

plethora of questions I had along the way: Jacob Brown, Jimmy Ling, Zhiyuan Ma, and Marat Musin. 
ACKNOWLEDGMENTS ................. ii

LIST OF TABLES $\ldots \ldots \ldots \ldots \ldots \ldots$ vi

LIST OF FIGURES $\ldots \ldots \ldots \ldots \ldots \ldots$ vii

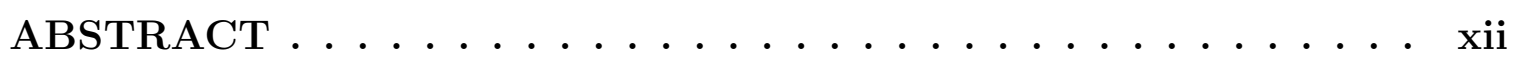

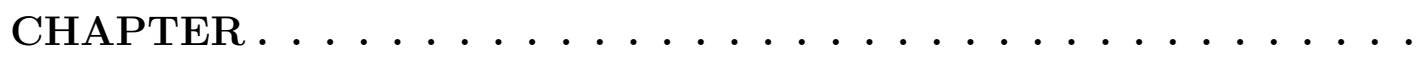

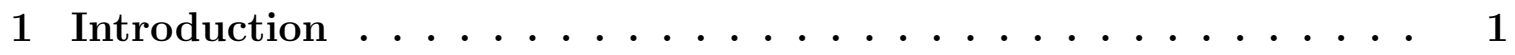

2 W4 Detected BCGs . . . . . . . . . . . . . . 5

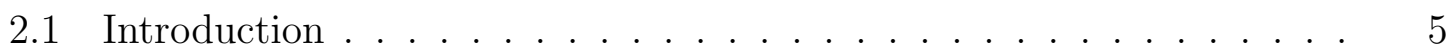

2.2 Data . . . . . . . . . . . . . . . . 6

2.2 .1 GMBCG Catalog . . . . . . . . . . . . . . . 6

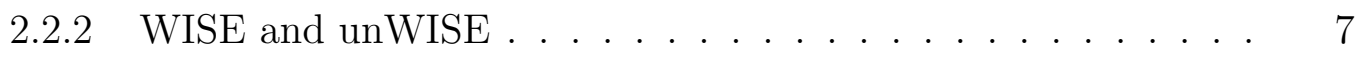

2.2 .3 Herschel data . . . . . . . . . . . . . . . . . . . 8

2.2 .4 Chandra Data . . . . . . . . . . . . . . . . . . 8

2.3 Sample of BCGs with strong mid-IR emission $\ldots \ldots \ldots \ldots$

2.3 .1 Initial Selection . . . . . . . . . . . . . . . . . . 10

2.3 .2 Sample Verification . . . . . . . . . . . . . . . . 11

2.3.3 General Properties and Subdivision of the Final Sample . . . 12

2.4 Data Analysis . . . . . . . . . . . . . . . . . . . . 13

2.4 .1 Possible AGN Hosts . . . . . . . . . . . . . . . . . 13

2.4 .2 Morphology . . . . . . . . . . . . . . . . . . 16

2.4 .3 Star Formation Rates . . . . . . . . . . . . . . . . . . 16

2.4 .4 Results . . . . . . . . . . . . . . . . . 20 
2.5 Cool-Core Clusters . . . . . . . . . . . . . . . . . . . . . . . 22

2.5.1 Archival Chandra Data . . . . . . . . . . . . . . . . . 23

$2.5 .2 \quad$ X-ray $c_{S B}$ Parameter . . . . . . . . . . . . . 23

2.5 .3 X-ray Spectral Fitting . . . . . . . . . . . . . . . 24

2.5.4 Lack of Cool-Core? . . . . . . . . . . . . . . . . 25

2.6 Discussion . . . . . . . . . . . . . . . . . . . . . . . . . . . . . 27

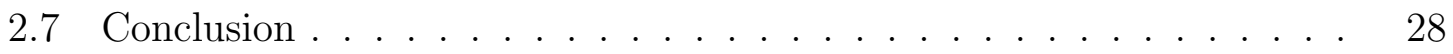

3 High-Mass Field Galaxies $\ldots \ldots \ldots \ldots$

3.1 Introduction . . . . . . . . . . . . . . . . 30

3.2 Data . . . . . . . . . . . . . . . . . . . . 31

3.2 .1 MPA-JHU Catalog . . . . . . . . . . . . . . . . 31

$3.2 .2 \quad$ SDSS DR12 . . . . . . . . . . . . . . . . . . 31

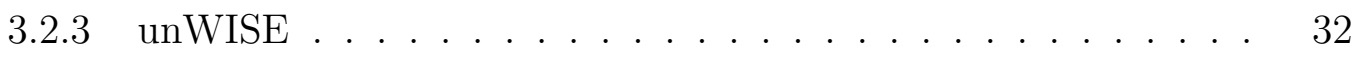

3.3 Selection of High Mass Galaxies with Strong Mid-IR Emission . . . . 32

3.3 .1 Sample Selection . . . . . . . . . . . . . . . 32

3.4 Data Analysis . . . . . . . . . . . . . . . . . . . 33

3.4 .1 Possible AGN Hosts . . . . . . . . . . . . . . . . . 33

3.4 .2 Morphology . . . . . . . . . . . . . . . . . . . 34

3.4.3 Star Formation Rates . . . . . . . . . . . . . . . . . . 35

3.5 Results . . . . . . . . . . . . . . . . . . 36

3.6 Comparison to W4BCGs . . . . . . . . . . . . . . . . . 38

3.7 Conclusion . . . . . . . . . . . . . . . . . . . . . . . . 40

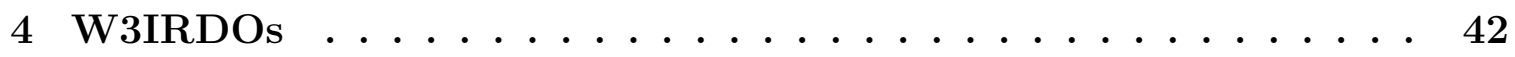

4.1 Why are these sources interesting? . . . . . . . . . . . . 43

4.2 Data . . . . . . . . . . . . . . . . . 44 
4.3 Sample Selection . . . . . . . . . . . . . . . . . . 44

4.4 Data Analysis . . . . . . . . . . . . . . . . . . . . . 45

4.5 Possible Causes . . . . . . . . . . . . . . . . . . 45

4.5 .1 Intrinsic $\ldots \ldots \ldots \ldots \ldots \ldots \ldots \ldots \ldots$

$4.5 .2 \quad$ Local . . . . . . . . . . . . . . . . . . . . . . . 47

4.6 Conclusions . . . . . . . . . . . . . . . . . . . . . . . . . . . . . . . . 49

5 Summary and concluding remarks $\ldots \ldots \ldots \ldots \ldots \ldots$

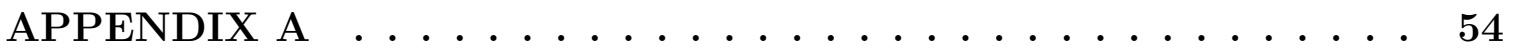

A Additional W4BCG Data ................. 54

A.1 W4BCGs in redMaPPer . . . . . . . . . . . . . 54

A.2 W4BCG Tables . . . . . . . . . . . . . 55

B FIR Templates for MPA-JHU Catalog . . . . . . . . . 59

B.1 SK07 vs. CE01 FIR Templates . . . . . . . . . . . . . . 59

BIBLIOGRAPHY .................. 62

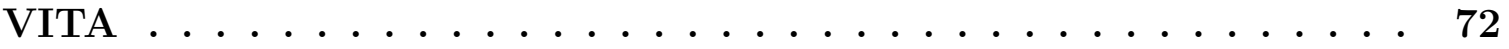




\section{LIST OF TABLES}

Table $\quad$ Page

2.1 Morphology of W4 detected BCGs based on SDSS $i$ ' images . . . . . 16

3.1 Possible AGN Hosts Based Upon WISE and BPT Diagnostics . . . . 34

3.2 Morphology of MPA-JHU Catalog Based Upon Galaxy Zoo . . . . . . 35

A.1 Summary of the Subset with Herschel Data . . . . . . . . . . 56

A.2 Summary of Available Archival Chandra Data . . . . . . . . . . . 57

A.3 X-Ray Spectral Fitting Results . . . . . . . . . . . . . . . 58 


\section{LIST OF FIGURES}

Figure

1.1 A representation of the color-magnitude diagram used to separate quiescent galaxies (red sequence) from star forming galaxies (blue cloud). Coutresy:Wikipedia/Joshua Schroeder . . . . . . . . . . . .

2.1 Examples showing WISE 4-band images of a BCG that is undetected in the W4 band (top) and one that is detected (bottom). These image cutouts are made from the unWISE products. The circles are centered on the reported SDSS positions, and are $10^{\prime \prime}$ in radius. . . . . . . .

2.2 Left: W4 (22 $\mu \mathrm{m})$ flux density distribution of the W4-detected BCGs (W4BCGs). Right: Normalized redshift distribution for the entire GMBCG catalog (red dashed line) and the subset of W4BCGs (blue solid line $) \ldots \ldots \ldots \ldots \ldots \ldots \ldots \ldots \ldots$

2.3 WISE color diagnostics of W4BCGs. The dashed line at $W 1-W 2=$ 0.8 mag separates AGN (red asterisks) and non-AGN (blue squares). Only 69 out of the total 389 W4BCGs are possible AGN hosts by this

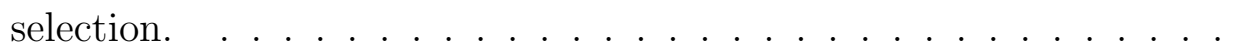


2.4 BPT diagnostics of W4BCGs. The dashed curve represents the criterion of Kauffman et al. 2003 that separates AGN-dominated objects (red pluses) and star-forming-dominated objects (blue asterisks). The black open squares ( 8 in total) indicate those that are also deemed to be AGN hosts based on the WISE color selection (see Fig. 2.3.) . .

2.5 SDSS $i^{\prime}$ band images illustrating the different morphology subsets. Left: A BCG with obvious merger properties. Middle: A BCG with a neighbor within $10^{\prime \prime}$ but no obvious merger properties. Right: A BCG with no other galaxies within a $10^{\prime \prime}$ radius. . . . . . . . . . .

2.6 Spectral energy distributions of the subsample of five W4BCGs that have Herschel data. The fitting of their stellar populations (shown by the blue curve) is the same as in the other W4BCGs, which incorporates both the SDSS photometry in optical and W1 and W2 in near-IR (see text for details). Two fitting schemes in the mid-to-far-IR regime are shown: one only using WISE data (dashed red) and one including Herschel data (solid yellow). The blue curve shows the SED fitting results of the stellar populations, which incorporate the SDSS photometry in optical and the WISE near-IR photometry in W1 and W2. The $\log \left(L_{I R}\right)$ value is displayed in the top left for each fitting along with the corresponding SFR (in $M_{\odot} / \mathrm{yr}$ ) in parenthesis. . . .

2.7 A histogram of the derived SFR for the entire W4BCGs sample (red solid line), the non-AGN host subset (green dot-dashed), and the subset with richness $\geq 15$ (blue dashed line; regardless of hosting AGN or

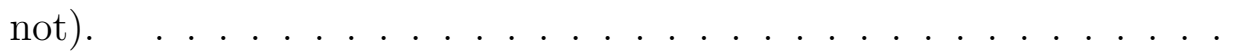


2.8 Left: Histogram distribution of the derived ages and masses for the "Rich" subset (richness $\geq 15$ ) among W4BCGs (solid blue) and the GMBCG catalog (dashed red) for $\mathrm{E}(\mathrm{B}-\mathrm{V})$ max values of: 0 (top), 0.3 (middle), and 0.5 (bottom). Right: Same as left except with the "Poor" subset $($ richness $<15) . \ldots \ldots \ldots \ldots$

2.9 A plot of $c_{S B}$ versus redshift of the 10 W4BCGs that have archival Chandra X-ray data. The dashed line at 0.075 separates non-coolcores from moderate cool cores. The dot-dashed line at 0.155 separates moderate cool-cores from strong cool-cores. Possible AGN hosts are color coded according to identification method: BPT in blue, WISE color in red, and both in purple. The three data points at $\mathrm{z}=0.14$ and the two at $\mathrm{z}=0.24$ are for $c_{S B}$ derived using different observations of the same object. The triangle shows the upper limit of the object that has no X-ray detection. . . . . . . . . . . . . . . .

2.10 The left panel shows J128.72875+55.57253 detected in the Chandra data with an exposure time of $9 \mathrm{ks}$ and $z_{\text {spec }}=0.24118$, while the right panel shows J125.63314+05.95189 not detected in the Chandra data with an exposure time of $8 \mathrm{ks}$ and $z_{\text {spec }}=0.081474$. The circles are centered on the reported SDSS positions and are $40 \mathrm{kpc}$ in size. . . .

3.1 Left: The WISE color-color plot for the MPA-JHU sample with the AGN dividing line at $\mathrm{W} 1-\mathrm{W} 2 \geq 0.8$. Right: The BPT diagram for the MPA-JHU sample with the Kauffman+03 line separating star-forming galaxies (below the line) from possible AGN (above the line). . . .

3.2 Left: The sSFR distribution for the entire sample (black line), the ellipitcal subset (red), and the spiral subset (cyan). Right: Same as on the left except only for the high-mass $\left(\log \left(\mathrm{M} / M_{\odot}\right) \geq 10.5\right)$ galaxies. 
3.3 The sSFR distribution for the W4 detected, high-mass galaxies. Colors are the same as previous figures. The peak of the distributions are emphasized by vertical lines: dot-dashed at $\sim-10$ for spiral and dashed at $\sim-9.75$ for elliptical. . . . . . . . . . . . . . . . .

3.4 Left: The stellar mass distribution for the W4 detected sample separated by elliptical and spiral morphology. Right: A normalized version of the figure on the left for a better comparison. . . . . . . . . . .

3.5 The SFR distribution for the W4 detected sample for the elliptical and spiral morphology. Vertical lines are located at the median value of the distribution: dot-dashed at $\sim 6$ for spiral and dashed at $\sim 12$ for

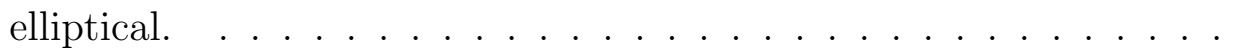

3.6 The redshift distribution for the MPA-JHU sample (blue solid) and the W4BCG catalog (red dashed). . . . . . . . . . . .

3.7 Left: The stellar mass distribution for the MPA-JHU sample (blue solid) and the W4BCG catalog (red dashed). Middle: The distribution of the $L_{i r}$ derived SFR for both samples. Right: The sSFR for both samples calculated from $L_{i r}$ based SFR. Vertical lines represent the median values for each sample. . . . . . . . . . . . . .

4.1 An example of a W3 dropout. SDSS ugriz bands are shown on top while WISE bands are shown on bottom. . . . . . . . . . . . . . . . . 42

4.2 The WISE response per photon for W1 (blue), W2 (green), W3 (orange) and W4(red). Courtesy: WISE . . . . . . . . . . . . . . 43

4.3 The distribution of the photometric redshifts of the W3IRDOs found using the BC03 models. . . . . . . . . . . . . . . .

4.4 The spectra of the closes ULIRG Arp 200 (black solid line) overplotted on the response curves for the WISE passbands. The prominent silicate absorption at $9.7 \mu \mathrm{m}$ falls within the W3 passband. Courtesy: WISE . 
4.5 The location of the W3IRDOs (red dots) projected onto the sky. A false color image from AKARI is used for illustration purposes. . . . . 47

4.6 The distribution of the Galactic latitude, $b$, for the W3IRDO sample. 48

4.7 A combined IRAS/COBE $100 \mu \mathrm{m}$ map of the sky illustrating the location of gas and dust. The filaments extending beyond the galactic plane are known as galactic cirrus. Courtesy: NASA . . . . . . . . . 49

A.1 Comparison of the derived SFR (left), stellar mass (middle), and age (right) for the W4BCGs from the GMBCG catalog (solid blue) and the redMaPPer catalog (red dashed), following the same SED fitting procedure as in $\S 2.4 .3$. The fit to the stellar population is based on $\mathrm{E}(\mathrm{B}-\mathrm{V})$ up to $0.3 \mathrm{mag}$. Each graph is normalized for easy comparison.

B.1 Left: The $L_{i r}$ estimate based upon the SK07 FIR templates versus redshift for the W4 detected MPA-JHU sample. A discontinuity appears at $\mathrm{z} \sim 0.13$. Right: Same as the figure on the left except for the W4BCG catalog. There is no obvious discontinuity present. . . . .

B.2 The $L_{i r}$ estimate based upon the CE01 FIR templates versus redshift for the W4 detected MPA-JHU sample. The discontinuity is no longer

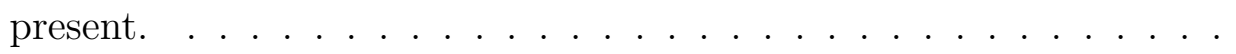

B.3 Left: A distribution of the difference between the $L_{i r}$ calculated by the SK07 templates and the CE01 templates for the W4BCG catalog. Right: The difference between SFR calculated using the $L_{i r}$ values from the figure on the left. . . . . . . . . . . . . . . 


\section{ABSTRACT}

We present a quantitative analysis of high-mass, low-z galaxies in order to investigate the 'downsizing' scenario of galaxy evolution. High-mass, low-z galaxies with ongoing star formation, antithetical to the 'downsizing' model, are identified using the $22 \mu \mathrm{m}$ data (W4 band) from the Wide-field Infrared Survey Explorer (WISE). A cluster and field sample is chosen to investigate any possible environmental effects. The cluster sample is based upon the GMBCG catalog, which contains 55,424 brightest cluster galaxies (BCGs) at $0.1 \lesssim z \lesssim 0.55$ identified in the Sloan Digital Sky Survey (SDSS). We identify 389 W4-detected BCGs (W4BCGs) that have median SFRs of $\sim 50 M_{\odot} / y r$ based upon their total IR luminosity $\left(L_{I R}\right)$, which is attributed to dust-enshrouded star formation. BCGs with such high SFRs are found in "coolcore" clusters and the star formation is thought to be fueled by a "cooling flow." Using Chandra X-ray data, it is shown that a subset of BCGs do reside within coolcores, but their mass deposition rates cannot account for the SFR. For comparison, a field sample is drawn from the Max-Planck Institute for Astrophysics - John Hopkins University (MPA-JHU) "value-added" SDSS DR7 catalog of spectrum measurements. A set of 1,244 high-mass, elliptical field galaxies within the same redshift range as the W4BCG catalog are identified for comparison. The median mass for the field sample is lower than the $\mathrm{W} 4 \mathrm{BCGs}\left(\log \left(\mathrm{M} / M_{\odot}\right)=10.9\right.$ and 11.2 respectively), as are their SFRs. However, the specific star formation rate (sSFR), the star formation rate per stellar mass, is comparable for both groups ( $\log (\mathrm{sSFR}) \sim-9.7)$. This possibly reveals that there is no environmental dependence on the sSFR for these W4-detected galaxies. While a possible mechanism responsible for the SFR was identified for the W4BCGs, the process responsible for the star formation in the field sample requires further investigation. 


\section{Chapter 1}

\section{Introduction}

Like most things, galaxies change with time. This process is known as galaxy evolution. A number of sources can affect how a galaxy evolves: environment, mass, age, metallicity, etc... By studying the properties of galaxies at varying redshifts, a picture begins to form that outlines the evolutionary tracks that galaxies follow. One such scenario postulated by Cowie el al. (1996) is known as 'downsizing.' [1] According to the 'downsizing' picture, the most massive galaxies formed first and the bulk of star formation shifts to less massive systems at lower redshifts. This implies that the most massive galaxies at low redshifts built up a majority of their stellar mass at an early time $(\mathrm{z}>1)$ before quenching occured, ultimately reducing the rate at which stars are formed. Therefore, local high-mass galaxies should appear to be passiveley evolving with little-to-no ongoing star formation. Indeed, this has been confirmed in a number of galaxy surveys.[2, 3, 4, 5, 6, 7]

In order to ease the study of 'downsizing,' galaxies can be separated into two categories: early-type galaxies (ETGs) and late-type galaxies (LTGs). ETGs have elliptical/spheroidal morphologies and are typically dominated by old stellar populations at low redshifts. Due to this dominant older stellar population, they appear red in optical wavelengths. In contrast to that, LTGs have spiral morphologies and, due 
to their young stellar population, appear blue in optical wavelengths. Based upon the 'downsizing' scenario and confirmed by observations, ETGs tend to be more massive than LTGs and have lower amounts of star formation at later epochs. However, no model is completely perfect, and there may exist a small sample of ETGs that exhibit high amounts of star formation at low redshifts. Exploring these outliers will help to further our understanding of galaxy evolution.

If we want to find such outliers, we need to identify galaxies with active star formation. As detailed above, old stellar populations appear red while young stellar populations are blue in optical. Capaitalizing upon these properties, galaxies can be separated into star forming and quiescent (little or no star formation) simply by analyzing their colors in optical bands. In practice, this sufficient to separate the two systems, resulting in what is known as the red sequence, primarily composed of quiescent ellipticals, the blue cloud, mostly made up of star-forming spirals, and the green valley, where star-forming ellipticals and quiescent spiral galaxies likely reside (see Fig. 1.1).

However, optical colors only reveal the visible or unobscured star formation. Most star formation occurs in areas of dense dust, where the dust facilitates the cooling of gas which condenses into forming stars. The dust scatters the bluer light (shorter wavelengths) from stars resulting in a reddening of a galaxy's color. This means, it is possible to underestimate or completely miss star formation that is obscured by thick dust.

Fortunately, the heated dust emits at longer wavelengths in the infrared (IR). The reprocessed starlight can be observed by various IR infrared observatories (e.g. Infrared Astronomy Satellite (IRAS), Wide-field Infrared Survey Explorer (WISE), Herschel, and Spitzer). Following the principal that the IR emission is from heated dust due to star formation activity, it is possible to convert the IR luminosity $\left(L_{i r}\right)$ into a star formation rate. Kennicutt 1998 found that the $L_{i r}$ measured from 8- 


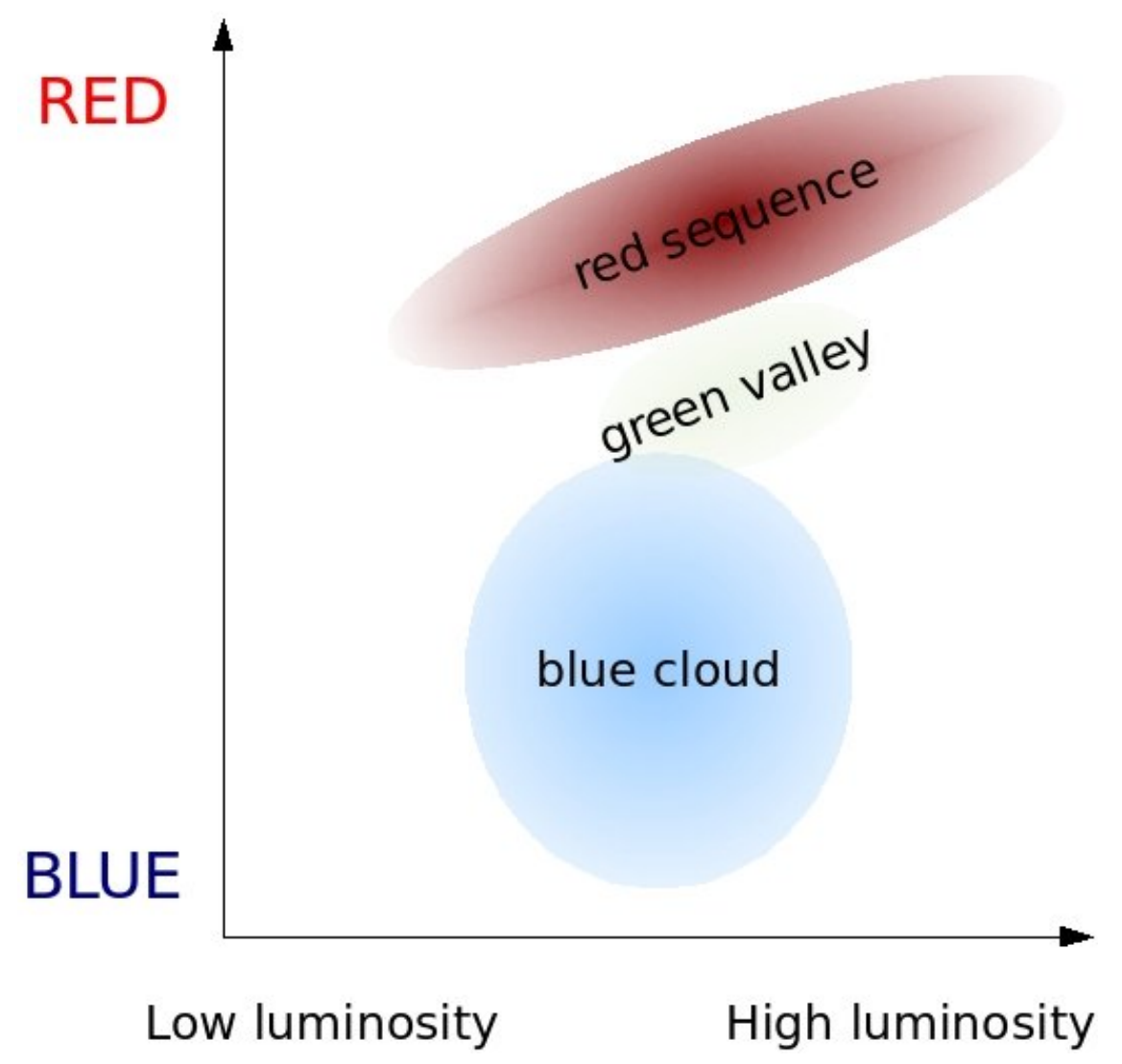

Figure 1.1: A representation of the color-magnitude diagram used to separate quiescent galaxies (red sequence) from star forming galaxies (blue cloud). Coutresy:Wikipedia/Joshua Schroeder

$1000 \mu \mathrm{m}$ provided a robust star formation estimate.[8] It has also been shown that a monochromatic (single-band) approach at $24 \mu \mathrm{m}$ is a good tracer to $L_{i r}$ and the star formation rate. $[9,10]$

In this research, we will search for high-mass, low-redshift galaxies that exhibit large amounts of star formation, in opposition to the 'downsizing' model. Instead of depending upon optical color to choose star-forming galaxies, which could miss heavily obscured star formation, clear detection in the WISE passband 4 (W4) at $22 \mu \mathrm{m}$ will be used as a proxy for star formation as explained in the previous paragraph. The first sample investigated is the most massive and reddest galaxies found within galaxy clusters. In order to investigate any environmental effects, a sample of high-mass galaxies is chosen from a catalog of field galaxies. As stated previously, the properties 
of these outliers within the context of the 'downsizing' model should improve our understanding of galaxy evolution. The results of this research is presented in the following sections, along with an investigation of a category of galaxies with unusual mid-IR properties. 


\section{Chapter 2}

\section{W4 Detected BCGs}

\section{$2.1 \quad$ Introduction}

A Brightest Cluster Galaxy (BCG), as the name implies, resides within a galaxy cluster and is its brightest member. BCGs are among the most luminous and the most massive galaxies in the low-redshift universe, usually have little ongoing star formation, and are dominated by old stellar populations.[11] It is believed that they have assembled most of their stellar masses before $z \sim 3$, and have been passively evolving ever since.[12] For this reason, they are among the so-called "red-and-dead" galaxy population.

BCGs being largely quiescent in the low-redshift universe is consistent with the general picture of "downsizing" galaxy evolution, where the bulk of the star formation activities in the universe shift from high mass galaxies to low mass ones as the universe evolves.[1] On the other hand, it has been known for over a decade that some BCGs at low redshifts still exhibit significant star formation. Such BCGs are in "coolcore" clusters, whose intra-cluster medium (ICM) has a temperature gradient such that materials can be funneled to the central region where the BCGs reside and 
presumably can fuel the observed star formation (see Donahue et al. 2015 for recent discussions).[13, 14, 15, 16, 17] However, it is unclear what fraction of BCGs still have ongoing star formation.

In this work, we present a systematic study of star-forming BCGs, using the data from the Sloan Digital Sky Survey (SDSS) and the Wide-field Infrared Survey Explorer (WISE). [18, 19] We make use of the GMBCG catalog of Hao et al. 2010 (hereafter Hao10), which is the largest BCG catalog available to date, and select those BCGs that potentially have strong ongoing star formation activities based on their properties in the mid-IR bands of WISE.[20] Our goal is to shed new light to the understanding of star-forming BCGs as a whole: how rare they are, how high their star formation rates (SFRs) can be, whether they have different properties in other aspects as compared to the vast majority of BCGs that are quiescent, and whether residing in "cool-core" clusters is a satisfactory explanation to their SFRs.

\subsection{Data}

The critical data sets used to select star-forming BCGs are the SDSS-based GMBCG catalog and the WISE all-sky survey data. In particular, we adopt the "un-blurred" version of the WISE data (also known as "unWISE") of Lang 2014 for this study.[21] A small number of such selected BCGs also have far-IR (FIR) data from Herschel or X-ray data from Chandra, which we used for further analysis. All these data are briefly described below.

\subsubsection{GMBCG Catalog}

The GMBCG Catalog consists of 55,424 rich galaxy clusters found by using the Gaussian Mixture Brightest Cluster Galaxy (GMBCG) algorithm on the SDSS data

in the seventh data release (DR7).[20] This algorithm detects clusters by identifying 
the BCG and the red sequence galaxies in its vicinity and calculating the clustering strength, a measure of the surface density of cluster galaxies at the BCG position. Hao10 apply this method to the Legacy Survey Area of SDSS DR7, which covers 7,300 $\mathrm{deg}^{2}$ of the North Galactic Cap and $740 \mathrm{deg}^{2}$ from three stripes in the South Galactic Cap, and obtain their cluster catalog across the redshift range $0.1 \lesssim z \lesssim 0.55$.

This GMBCG catalog contains the positions of the identified BCGs along with their redshifts and photometry. The redshifts are either spectroscopic redshifts $(\sim 20,000$ objects) or photometric redshifts (see Hao10 for details).

\subsubsection{WISE and unWISE}

The nominal WISE mission mapped the entire sky in 2010 in four near-to-mid-IR bands, namely, W1, W2, W3, and W4, whose central wavelengths are 3.4, 4.6, 12, and $22 \mu \mathrm{m}$, respectively. The spatial resolutions in these four bands are 6.1", 6.4", $6.5^{\prime \prime}$, and $12.0^{\prime \prime}$, respectively. The nominal $5 \sigma$ limits in these bands are 0.068, 0.098, 0.86 and $5.4 \mathrm{mJy}$, respectively.[19]

The offically released images of WISE ("AllWISE") were intentionally convolved by the point spread functions (PSFs) during the co-adding process. While this process is appropriate for isolated point sources, it reduces the resolution of the images and thus exacerbates the blending problem. To remedy this problem, unWISE ${ }^{1}$ "un-blurs" these images to produce the final stacks that preserve the native spatial resolutions.[21]

Along with the un-blurred images, unWISE also provides a catalog of WISE photometry based on "forced photometry" using $~ 400$ million SDSS DR10 objects as the morphological templates to fit the WISE source light profiles.[22] Since the GMBCG catalog is based upon the same SDSS data (albeit in an earlier data release), all of our objects appear in the unWISE forced photometry catalog. Therefore, we adopted

\footnotetext{
${ }^{1}$ http://unwise.me
} 
the unWISE images for visual verification and its forced photometry for quantitative analysis.

\subsubsection{Herschel data}

In order to further study the star formation properties of the selected BCGs, we also used the archival FIR data taken by the Spectral and Photometric Imaging Receiver (SPIRE) on Herschel Space Observatory.[23, 24] While only a small number of objects have these SPIRE data, they offer a valuable reference that is will be detailed in $§ 2.4 .3$.

Specifically, we made use of the SPIRE three-band (250, 350 and $500 \mu \mathrm{m})$ photometry from the following Herschel very wide-field surveys whose SPIRE data are now publicly available, namely, the Herschel Multi-tiered Extragalactic Survey (HerMES), the Herschel Stripe 82 Survey (HerS) and the Herschel Astrophysical Terahertz Large Area Survey (H-ATLAS).[25, 26, 27, 28, 29, 30, 31, 32] Both HerMES and H-ATLAS have catalogs available that include flux measurements. For the HerS data, we measured the source flux on the SPIRE images using HIPE (Herschel Interactice Processing Environment) following the procedure for source extraction and photometry outlined in the SPIRE data manual ${ }^{2}$.[33] In total, these surveys cover $340 \mathrm{deg}^{2}$.

\subsubsection{Chandra Data}

In order to investigate possible cool-core properties of our sample, X-ray data is necessary. Therefore, we used the public Chandra X-ray data provided by the Chandra Data Archive. Both Primary and Secondary products were retrieved for each available observation. These data provide a spatial resolution of $0.5^{\prime \prime}$ and cover an energy range of $0.1-10 \mathrm{keV}$.

\footnotetext{
${ }^{2}$ http://herschel.esac.esa.int/hcss-doc-12.0/
} 
Standard data processing was carried out starting from the level 1 event files using CIAO 4.8.2 (Chandra Interactive Analysis of Observations) with CALDB 4.7.0 of the Chandra Calibration Database.[34] The reprocessing script chandra_repro was used to reprocess the data and create level 2 event files. When observations were taken in the VFAINT mode, the parameter check_vf_pha was set to "yes" in order to remove background events likely caused by cosmic rays. Background estimates were taken in the same field away from the central X-ray peak and clear of any other X-ray sources.

For X-ray spectra, we followed a procedure similar to that of Molendi et al. 2016 (hereafter Mol16).[35] The X-ray spectra for the BCG were processed from the level 2 event files using specextract in CIAO. A $40 \mathrm{kpc}$ region centered on the BCG was chosen for the extraction. There could be a complication in this analysis if the BCG is an X-ray AGN, in which case the X-ray spectrum might be dominated by the AGN rather than the heated ICM. To solve this potential problem, we performed a separate analysis by following Mol16 and masking the central region. Unlike in Mol16 where a circular region of $2^{\prime \prime}$ in radius is masked, we chose to only mask out the inner $2 \mathrm{kpc}$ of the BCG, as choosing a global value of $2^{\prime \prime}$ would result in masking out the bulk of X-ray flux for some of our sources. Background spectra were also processed at up to three different regions away from the X-ray peak and any other X-ray sources.

\subsection{Sample of BCGs with strong mid-IR emission}

We searched for BCGs with ongoing star formation by identifying those that have secure mid-IR detections in the WISE W4-band at $22 \mu \mathrm{m}$. This will reveal dustembedded star formation in BCGs, and thus is complementary to the method that aims at identifying unobscured star formation through UV emissions, such as some of those reported by Donahue et al. 2015.[17] Here we describe our sample in detail. 

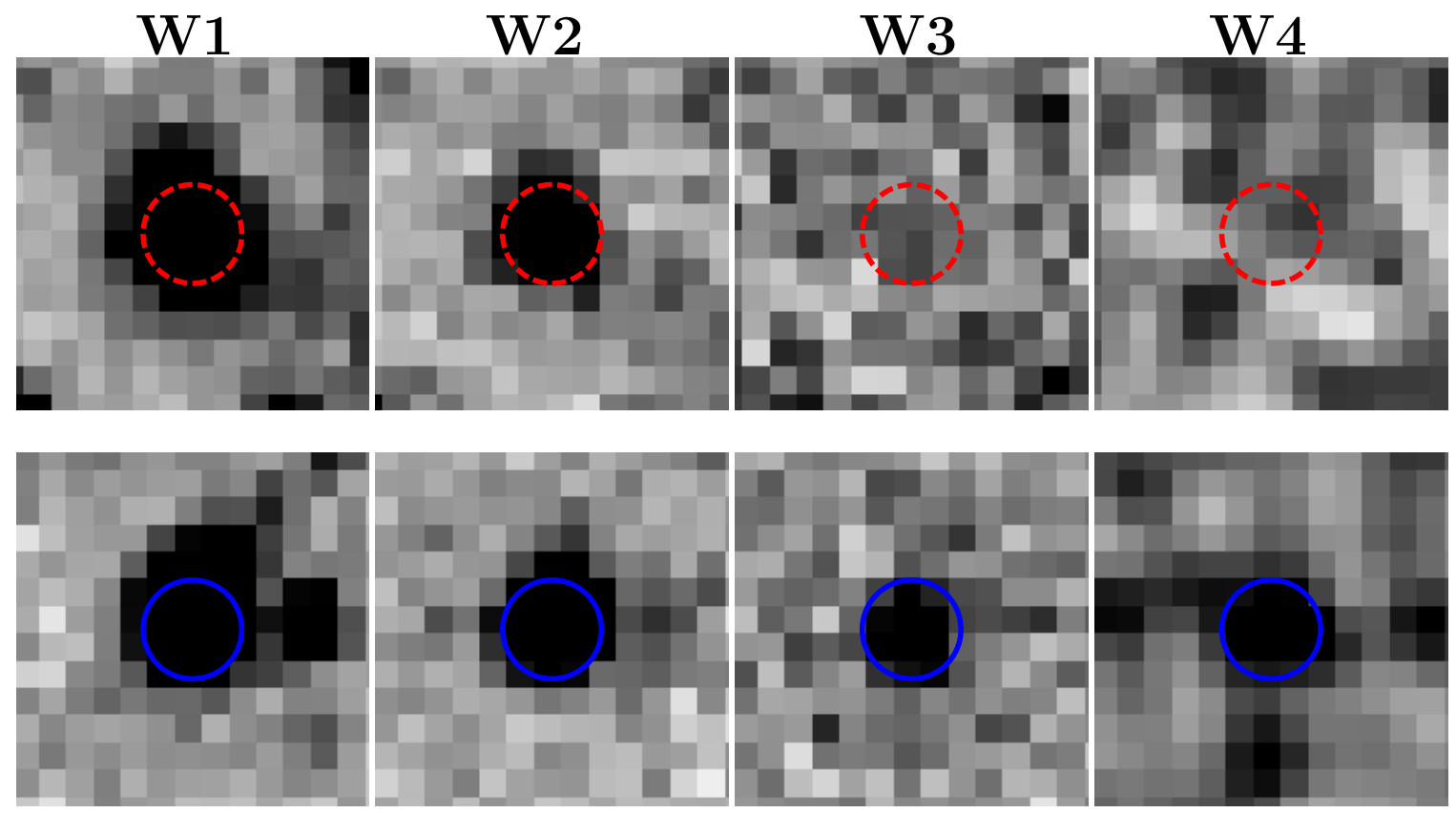

Figure 2.1: Examples showing WISE 4-band images of a BCG that is undetected in the W4 band (top) and one that is detected (bottom). These image cutouts are made from the unWISE products. The circles are centered on the reported SDSS positions, and are $10^{\prime \prime}$ in radius.

\subsubsection{Initial Selection}

To construct a catalog of possible sources that have W4 detection, the GMBCG catalog was cross-matched with the unWISE forced photometry catalog using a matching radius of $5^{\prime \prime}$, which is slightly less than half the spatial resolution of W4. We further required that a matched object should have $S / N \geq 5$ in W4 as reported in the unWISE SDSS forced photometry catalog. This resulted in 1,323 BCGs in our initial sample.

To ensure the sample robustness, we visually inspected the images of all these initial candidates. We found that a large number of the reported W4-detections were actually false-positives due to various reasons, such as image defects, noise spikes, artifacts produced by a bright neighbor, etc. After rejecting these contaminators, 458 BCGs survived. As an example, Fig. 2.1 shows the WISE image stamps of one that is not detected in W4 and one with a real W4-detection. 


\subsubsection{Sample Verification}

Obviously, W4-detected BCGs are only a small fraction of the entire GMBCG sample. Therefore, we must consider possible contamination to the GMBCG sample, or in other words, whether these W4-detected objects derived from the GMBCG sample are BCGs at all. To address this question, we further verified the legitimacy of these 458 candidate objects on a one-by-one basis. This verification was to decide whether a candidate is in a cluster environment, and if yes, whether it is the BCG of the cluster. Our intention was not to invent a new cluster finding algorithm, but to perform an independent "sanity check" on the claimed BCGs.

The verification consisted of two steps. First, we worked under the assumption that the photometric redshifts that the GMBCG catalog relies on are accurate enough for its purpose. We used the SDSS DR7 data, the same as what the GMBCG catalog is based. For each candidate BCG, we retrieved the objects within a $3^{\prime}$ radius around it, and retained only those whose photometric redshifts (as reported in the SDSS DR7) were within \pm 0.02 of the redshift of the candidate BCG (as quoted in the GMBCG catalog and is the same as in the SDSS DR7). The retained objects were considered as the members of the candidate cluster. This redshift range was adopted because it is the reported accuracy $(1 \sigma)$ of the SDSS DR7 photometric redshifts. We then constructed the $i$ vs. $(g-r)$ color-magnitude diagram, and checked whether we could see a "ridge line" indicative of a red sequence. If a red sequence was seen, we checked whether the current candidate BCG was the correct identification of BCG, i.e., whether it was the brightest one (in $i$-band) among all members.

After this step, we confirmed that 383 objects survived. Among the 75 dubious cases, four could hardly be called clusters because they only had a few members $(<8)$, and thus must be rejected. These four sources were at the high-redshift end of the catalog. One other case was a misidentification, and actually must be part of Abell 1689 (whose BCG is already in the W4BCG sample) and thus must also be removed. 

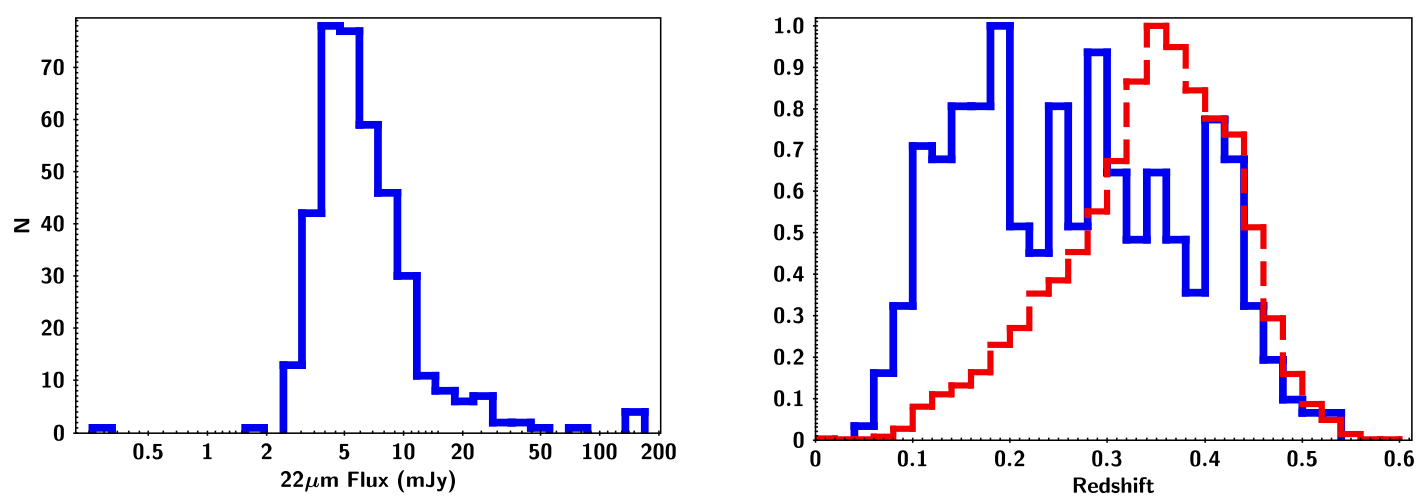

Figure 2.2: Left: W4 $(22 \mu \mathrm{m})$ flux density distribution of the W4-detected BCGs (W4BCGs). Right: Normalized redshift distribution for the entire GMBCG catalog (red dashed line) and the subset of W4BCGs (blue solid line).

The other 70 objects were in clusters with a clear red sequence, however they were in fact not the BCGs. Therefore, we identified the "new" BCG for each of these 70 cases by finding the brightest member, and conducted all the previous steps reported above on these "new" BCGs. Of all these 70 objects, only six have reliable W4 detections. We included these six objects into our sample, and thus our final sample consists of 389 objects in total.

\subsubsection{General Properties and Subdivision of the Final Sam- ple}

Fig. 2.2 shows the distributions of their W4 flux densities and redshifts. At these redshifts, the W4 emissions are still in the rest-frame mid-IR, and must be originated from heated dust instead of stellar continuum. These W4-detected BCGs (hereafter "W4BCGs") comprise a special population at odd with the general picture of BCGs that they are old, passively evolving galaxies. Therefore, we aim to understand the nature of these exceptions.

In order to investigate whether the occurrence of W4BCG could be dependent of the cluster richness, we divide our final W4BCG sample into two categories based on 


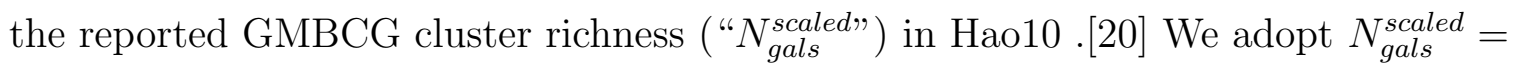
15 as the criterion, and refer to those clusters with $N_{\text {gals }}^{\text {scaled }} \geq 15$ as "rich" clusters and those with $N_{\text {gals }}^{\text {scaled }}<15$ as "poor" clusters. The rich clusters are $28 \%$ of the entire GMBCG sample, while the poor clusters make of $72 \%$. The corresponding W4BCGs are subsequently divided into the W4BCG-R (108 objects, or $27.8 \%$ of the total 389 W4BCGs) and the W4BCG-P (281 objects, or 72.2\%) subsamples, respectively.

\subsection{Data Analysis}

There are two possible causes to the heated dust emissions of these W4BCGs in the mid-IR, namely, active ongoing star formation or AGN activities. In this section, we investigate which of these two mechanisms is the more probable cause.

\subsubsection{Possible AGN Hosts}

To understand whether any of our W4BCGs could possibly host an AGN, we performed two diagnostics, which are based on the WISE color selection and the BPT diagram method, respectively. We note that being diagnosed as an AGN host by either method does not necessarily mean that the mid-IR emission in W4 must be dominated by AGN heating. However, if we do not find any AGN activity by either method, it is very plausible that the mid-IR emission is mainly driven by the heating of star formation.

\section{WISE Color Diagnostics}

Using a W1-W2 versus W2-W3 WISE color-color plot has been shown to be an effective method to identify AGN.[36, 37, 38, 39] Furthermore, it has been demonstrated that a single color criterion of $W 1-W 2 \geq 0.8 \mathrm{mag}$ (in Vega system) provides a 


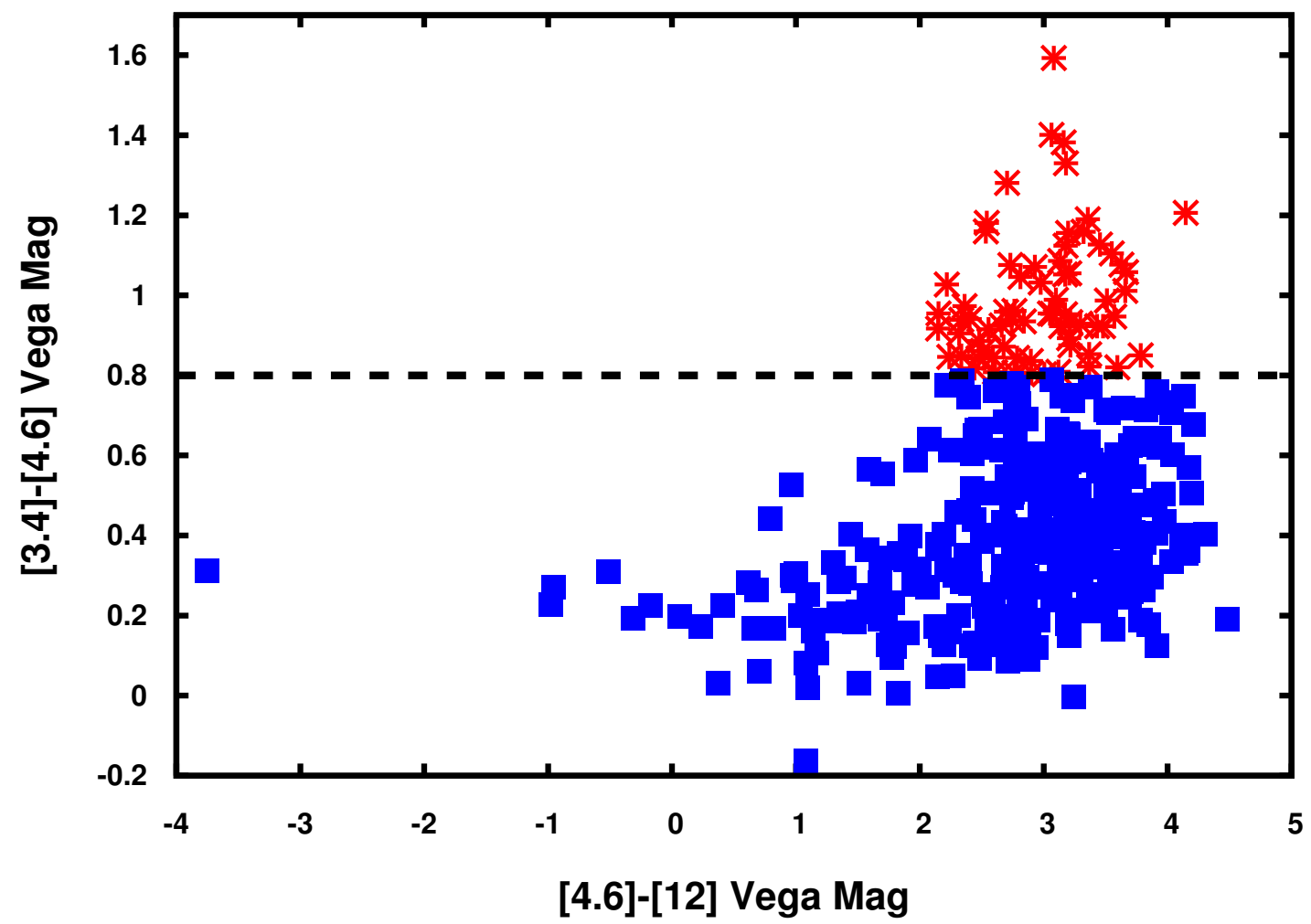

Figure 2.3: WISE color diagnostics of W4BCGs. The dashed line at $W 1-W 2=$ 0.8 mag separates AGN (red asterisks) and non-AGN (blue squares). Only 69 out of the total 389 W4BCGs are possible AGN hosts by this selection.

robust selection of AGN.[38, 39] We adopted this latter method in our analysis, and the result is shown in Fig 2.3. We find that only 69 out of the total 389 W4BCGs and 12 of the 108 W4BCG-Rs satisfy this criterion (17.7\% and $11.1 \%$ respectively), or in other words, most W4BCGs should be dominated by starbursts.

\section{BPT Diagram}

BPT diagrams, named after Baldwin, Phillips, and Terlevich, are a set of diagnostic diagrams using emission lines to determine the ionization mechanism of nebular gas.[40] The most commonly used diagram is [OIII] $5007 / \mathrm{H} \beta$ versus [NII] $6584 / \mathrm{H} \alpha$, which is what we used in our analysis. Various dividing curves have been proposed to separate AGN from star-forming galaxies.[41, 42] The curve of Kauffman et al. 


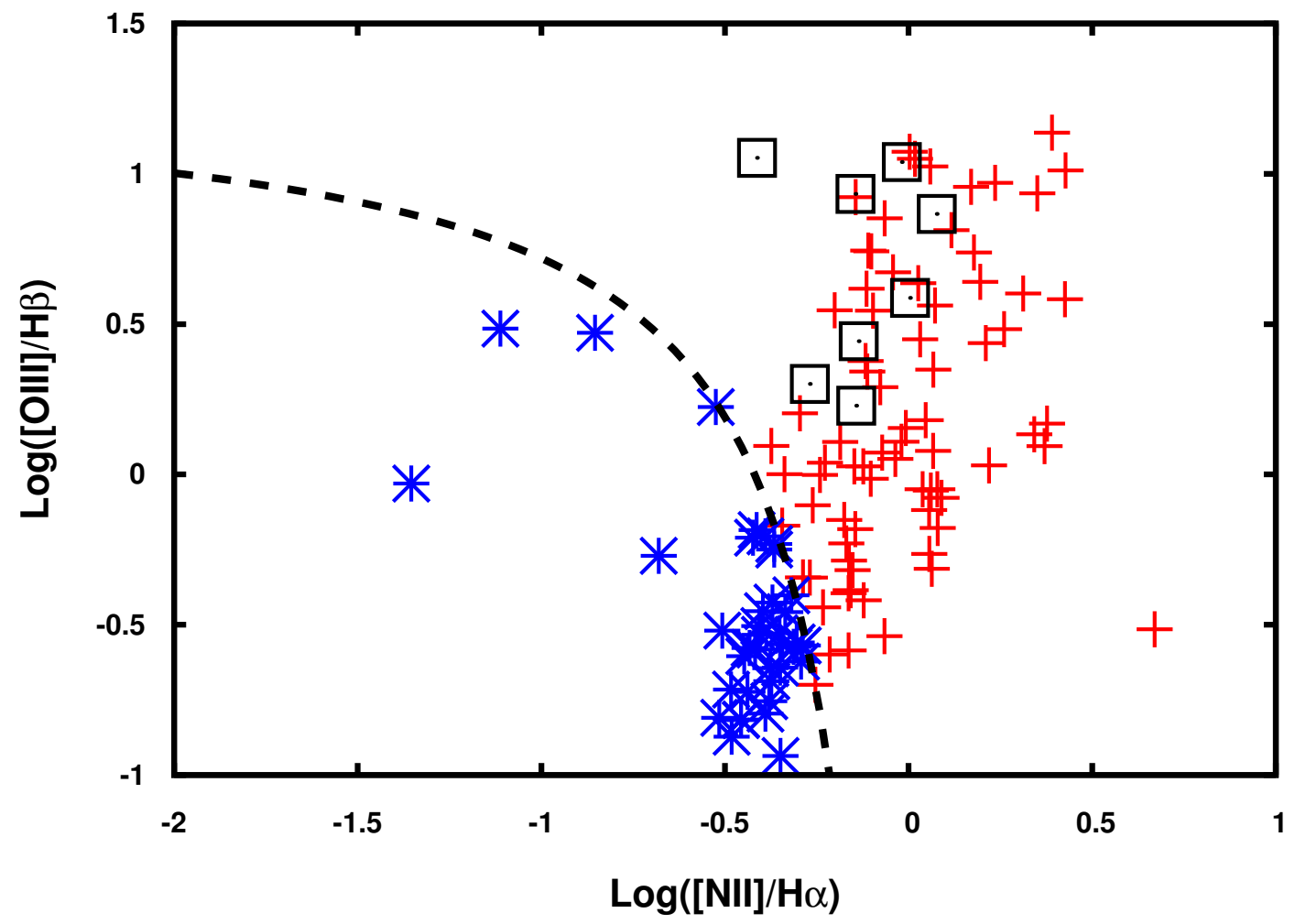

Figure 2.4: BPT diagnostics of W4BCGs. The dashed curve represents the criterion of Kauffman et al. 2003 that separates AGN-dominated objects (red pluses) and star-forming-dominated objects (blue asterisks). The black open squares (8 in total) indicate those that are also deemed to be AGN hosts based on the WISE color selection (see Fig. 2.3.)

2003, which is shown in Eq. 2.1 below, is the most aggressive in selecting AGN, and hence we adopted this selection criterion in order to be conservative in attributing W4BCGs to starburst:

$$
\log ([O I I I] / H \beta)=0.61 /(\log ([N I I] / H \alpha)-0.05)+1.3
$$

For emission line measurements, we used the MPA-JHU(Max-Planck Institute for Astrophysics - John Hopkins University) "value-added" DR7 catalog of spectrum measurements based on the SDSS DR7 data. [43, 44, 45] A cross-match to the MPAJHU catalog (within a radius of 5 " of the GMBCG reported position) resulted in 123 objects with emission line measurements for all four lines needed for the BPT 15 
Table 2.1: Morphology of W4 detected BCGs based on SDSS i' images

\begin{tabular}{lc} 
Type & Count \\
\hline Merger & 69 \\
Close Neighbor & 145 \\
Single & 175
\end{tabular}

diagnostic, which is shown in Fig 2.4. Within this subsample of 118 W4BCGs, 84 are deemed to host AGN (29 are W4BCG-R).

\subsubsection{Morphology}

The morphologies of the W4BCGs may provide additional information to reveal the nature of their mid-IR emissions. In particular, we are interested in understanding whether merger could be relevant, regardless of the exact heating source being AGN activities or star formation. For this purpose, the SDSS $i^{\prime}$ band images were examined. The W4BCGs were then divided into three different categories: "Merger", where a recent or ongoing merger is evident as shown by disturbed morphology; "Close Neighbor", where there is no clear sign of merger but there is at least one galaxy within $10^{\prime \prime}$ (even though this could be due to projection by chance); and "Single", where there is no sign of merger and no other galaxies within $10^{\prime \prime}$ to the SDSS depth. Some examples are shown in Fig. 2.5. We note that $10^{\prime \prime}$ corresponds to $18-60 \mathrm{kpc}$ at the redshifts of the W4BCGs.

The statistics are listed in Table 2.1, which shows that the majority of W4BCGs do not exhibit obvious merger properties. Therefore, it is safe to conclude that the mid-IR emission of a W4BCG is independent of whether it is interacting with others.

\subsubsection{Star Formation Rates}

The analysis above clearly shows that most W4BCGs are not AGN hosts, and hence their mid-IR emission can only come from dust heated by strong star formation. 

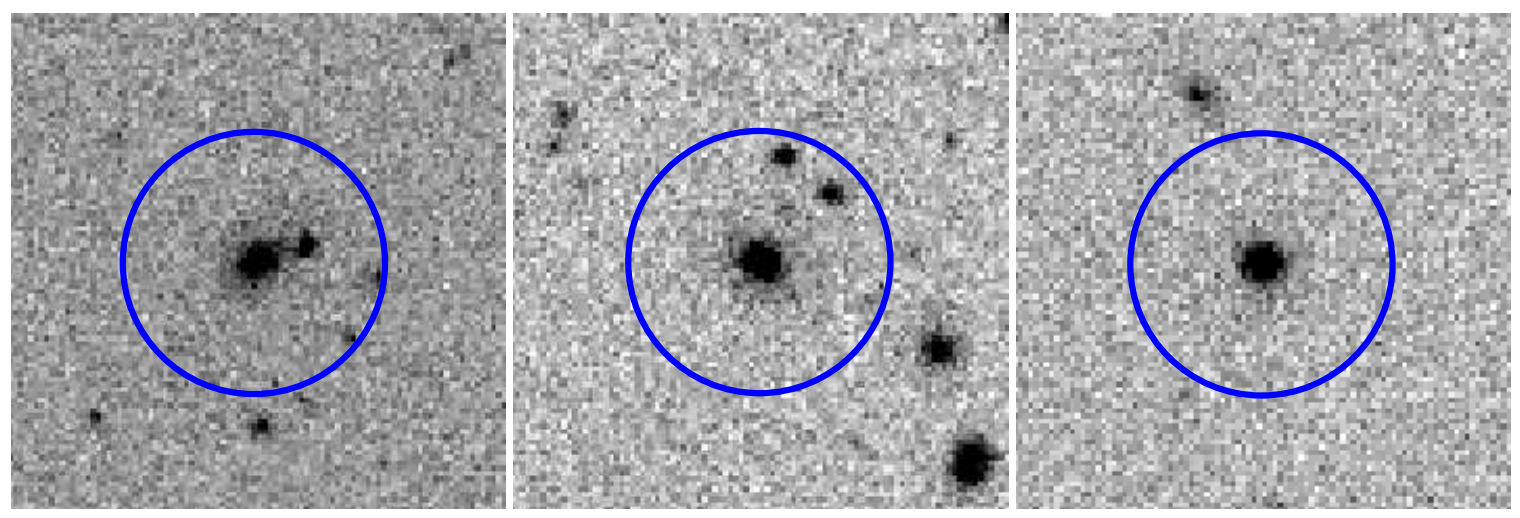

Figure 2.5: SDSS $i^{\prime}$ band images illustrating the different morphology subsets. Left: A BCG with obvious merger properties. Middle: A BCG with a neighbor within $10^{\prime \prime}$ but no obvious merger properties. Right: A BCG with no other galaxies within a $10^{\prime \prime}$ radius.

Again, this is contrary to the general picture that BCGs are "red-and-dead" galaxies that have ceased their star formation long ago. This also leads to the question whether the mid-IR emissions of those AGN-hosting W4BCGs are due to AGN heating at all, as our data currently available cannot provide an unambiguous answer. In this section, we attempt to derive the star formation rates of the W4BCGs as a whole, assuming that the AGN contribution to their mid-IR emissions is negligible. In our later discussion, we examine whether this assumption is reasonable.

\section{SED Fitting}

A common method to derive IR-based SFR is to calculate the total IR luminosity $\left(L_{I R}\right)$ over the conventional range of $8-1000 \mu \mathrm{m}$ and then to infer the SFR by using the relation of Kennicutt 1998 as follows [8]:

$$
S F R\left[M_{\odot} / y r\right]=1.0 \times 10^{-10} L_{I R}\left[L_{\odot}\right]
$$

We note that the coefficient in the above equation is after adjusting to a Chabrier initial mass function (IMF) and the derived SFR is a factor of 1.7 smaller than in case of using a Salpeter IMF.[46, 47] 
$L_{I R}$ can be calculated by fitting the spectral energy distributions (SEDs) to appropriate templates of dusty star forming galaxies.[48, 49, 50] In our case, W3 and W4 can be used for this purpose. We are also interested in the properties of the stellar populations in the W4BCGs, such as stellar mass and age, which can be derived by fitting the SEDs at the bluer wavelengths to stellar population synthesis models. Therefore, we utilized LePhare, which is capable of fitting both the stellar populations and the heated dust components at the same time.[51, 52]

Our input SEDs were constructed using the SDSS DR7 photometry in conjunction with the unWISE photometry (as described in $\S 2.2 .2$ ) to cover the optical to midIR regime. The heated dust emission was fit to the template of Siebenmorgen \& Krügel (2007; SK07), and the fit was confined to the W3 and W4 bands only. For the stellar component, the fit included the five SDSS bands as well as the W1 and W2 bands. LePhare treats the transition of stellar emission and heated dust emission in a consistent manner; i.e., the contribution of the dust emission template to the bands bluer than W3 (or that of the stellar emission template to the bands redder than W2), albeit small, is still considered during the simultaneous fit of the two components. The stellar component was fit to the stellar population synthesis models of Bruzual \& Charlot (2003; hereafter BC03).[53] We adopted the BC03 models of solar metallicity and the Chabrier IMF, and used a series of exponentially declining star formation histories with $\tau$ ranging from 1 Myr to $20 \mathrm{Gyr}$. We note that we chose solar metallicity because we do not have any constraint on the metallicities of these objects, and the solar metallicity is the most widely adopted value throughout the literature in such case. The models were allowed to be reddened by dust following the Calzetti's law, with the reddening color excess value, E(B-V), allowed to vary over three ranges: from 0 up to $0.5 \mathrm{mag}$, from 0 up to $0.3 \mathrm{mag}$, and fix to zero (i.e., no reddening).[54] While the W4BCGs have very dusty star-forming regions, their exposed stellar populations as seen in optical-to-near-IR are not necessarily dusty. 


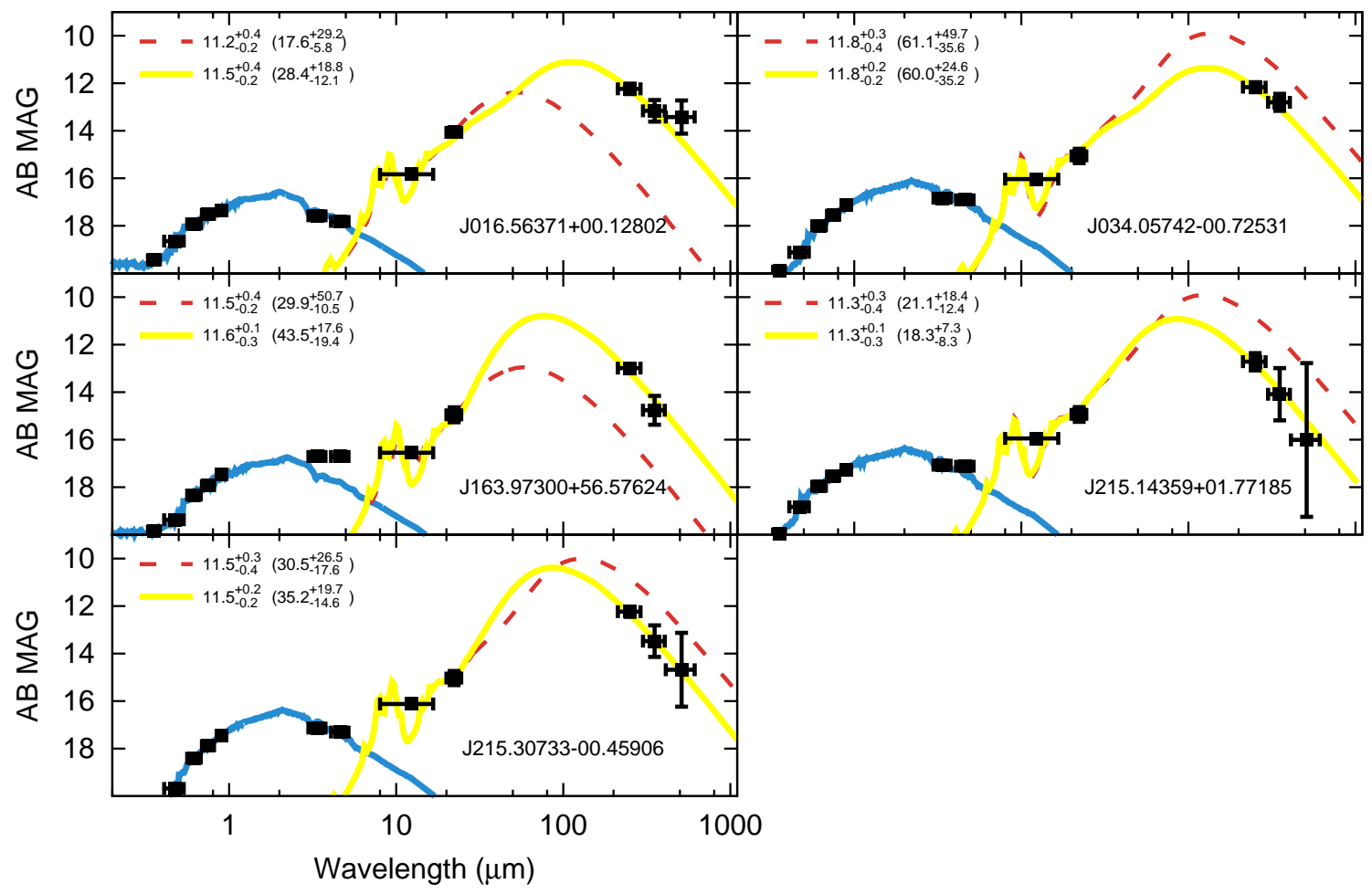

Figure 2.6: Spectral energy distributions of the subsample of five W4BCGs that have Herschel data. The fitting of their stellar populations (shown by the blue curve) is the same as in the other W4BCGs, which incorporates both the SDSS photometry in optical and W1 and W2 in near-IR (see text for details). Two fitting schemes in the mid-to-far-IR regime are shown: one only using WISE data (dashed red) and one including Herschel data (solid yellow). The blue curve shows the SED fitting results of the stellar populations, which incorporate the SDSS photometry in optical and the WISE near-IR photometry in W1 and W2. The $\log \left(L_{I R}\right)$ value is displayed in the top left for each fitting along with the corresponding SFR (in $M_{\odot} / \mathrm{yr}$ ) in parenthesis.

As the reddening parameter and the age of the stellar population are degenerated, we tested these three different choices to investigate the impact of different reddening values to the derived ages. Redshifts were fixed to those provided by the GMBCG Catalog.

\section{Far-IR Constraint from Herschel}

As W3 and W4 only sample a small mid-IR window of the entire rest-frame 8-1000 $\mu \mathrm{m}$ range, it could be a concern whether they can accurately "anchor" the fitting tem- 
plates to derive $L_{I R}$. A large number of practices in the literature have shown that one or two mid-IR bands indeed can derive $L_{I R}$ reasonably well, except that in the very high luminosity range such results tend to overestimate the true $L_{I R}$ (see e.g., Elbaz et al. 2011 and the references therein).[48, 55, 56, 57, 58] In order to check how well our derivation of $L_{I R}$ above can be, we tested a few objects that also have FIR SPIRE data as described in $\S 2.2 .4$, which samples the peak of the dust emissions and thus offers the most reliable derivation of $L_{I R}$ to date.

Following the same procedure of Ma \& Yan 2015, we found secure SPIRE counterparts within a matching radius of $3^{\prime \prime}$ for five W4BCGs: 2 in HerS, 1 in HerMES, and 2 in H-ATLAS.[59] A summary of the data is given in Table A.1. We ran LePhare to fit the SEDs of these objects as before, but with the SPIRE photometry added. Fig. 2.6 shows their SED fitting results. For these five objects, we find that the derived median $L_{I R}$ values with and without the inclusion of the SPIRE data differ by $\sim 0.1$ dex on average and 0.3 dex at most. Therefore, we believe that using $\mathrm{W} 3$ and W4 photometry to derive total $L_{I R}$ based on starburst templates (as in §2.4.3) is applicable.

\subsubsection{Results}

Applying Eq. 2.2 to our sample of 389 W4BCGs results in SFRs ranging from a few to $\sim 1000 M_{\odot} / y r$ (Fig. 2.7). The median $L_{I R}$ is $5 \times 10^{11} L_{\odot}$ (or SFR $\left.\sim 50 M_{\odot} / y r\right)$, and $27 \%$ of the whole sample has $L_{I R}>10^{12} L_{\odot}\left(\right.$ or SFR $\left.>100 M_{\odot} / y r\right)$. The statistics largely remain the same even if we only look at W4BCG-Rs or W4BCG-Ps, or if we remove any possible AGN hosts from the sample. Obviously, the W4BCGs are not "dead", i.e., they are not simply passively evolving like the BCG majority at low redshifts.

In addition to $L_{I R}$, SED fitting also derives stellar mass and age for each object. To check whether these W4BCGs have different stellar population properties as compared 


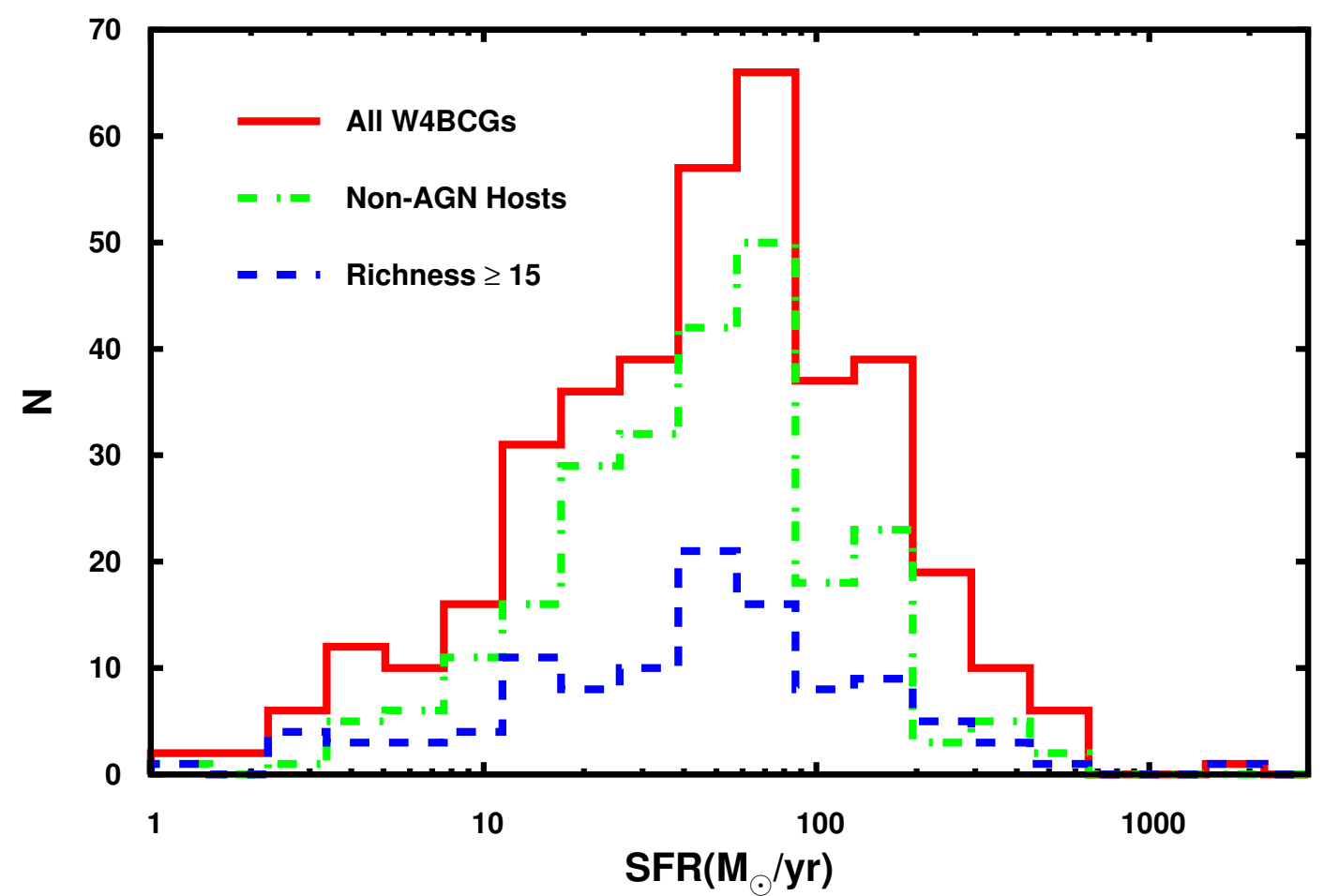

Figure 2.7: A histogram of the derived SFR for the entire W4BCGs sample (red solid line), the non-AGN host subset (green dot-dashed), and the subset with richness $\geq 15$ (blue dashed line; regardless of hosting AGN or not).

to the BCG majority, we performed SED fitting for all the non-W4BCGs from the entire GMBCG set in the same way as described in §4.3.1. The SEDs were based on the SDSS photometry and the unWISE photometry in W1 and W2. Fig. 2.8 compares the stellar mass and the age distributions of the W4BCGs to those of the non-W4BCGs. As expected from the reddening-age degeneracy, the ages of the W4BCGs show somewhat different trends with respect to the non-W4BCGs under different choices of allowed reddening range. This is further complicated by the fact that our SED fitting templates are fixed to solar metallicity. There is also an agemetallicity degeneracy in SED fitting, in the sense that adopting a lower metallicity could result in an older age. Therefore, no definite difference in trend can be claimed regarding the age comparison. The differences in stellar mass, on the other hand, show less variation in the trends under the three choices of allowed reddening ranges. 
Rich

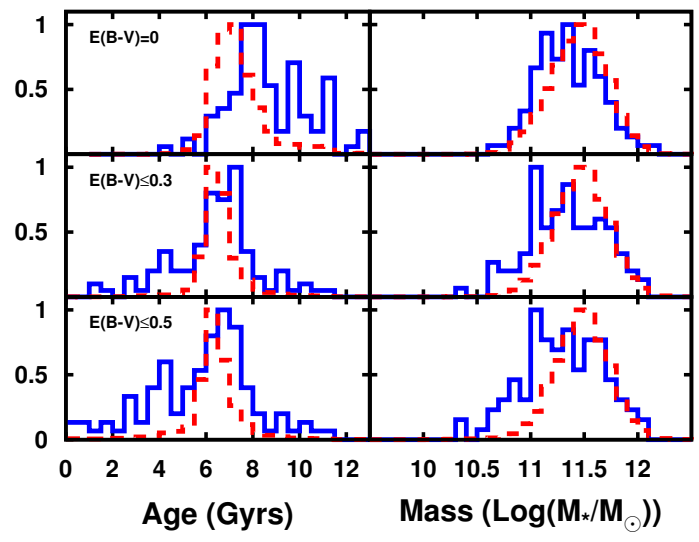

Poor

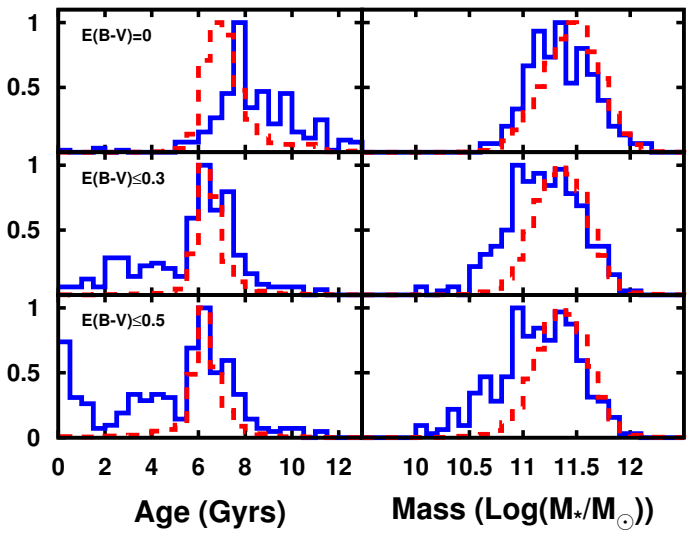

Figure 2.8: Left: Histogram distribution of the derived ages and masses for the "Rich" subset (richness $\geq 15$ ) among W4BCGs (solid blue) and the GMBCG catalog (dashed red) for E(B-V) max values of: 0 (top), 0.3 (middle), and 0.5 (bottom). Right: Same as left except with the "Poor" subset (richness $<15$ ).

Overall speaking, we believe that the W4BCGs do not show obvious differences as compared to the non-W4BCGs in mass and age of their exposed stellar populations.

\subsection{Cool-Core Clusters}

As mentioned in $\S 1$, it has been reported in the literature that some BCGs do exhibit ongoing star formation. While their SFR triggering mechanism is unclear, they are believed to reside in "cool-core" clusters. [60, 13, 14, 61, 15, 62, 17, 63, 35] Most of these previously reported star-forming BCGs in cool-core clusters have much lower SFR as compared to the bulk of our W4BCGs, with the most notable exception of the BCG in the Phoenix cluster at $z=0.597$, which has $S F R \sim 450 M_{\odot} / y r$ (after scaling to the Chabrier IMF).[64, 65, 66] In this section, we consider whether our W4BCGs reside in cool-core clusters (hereafter "CC clusters") as well, which can be determined by analyzing X-ray data. 


\subsubsection{Archival Chandra Data}

We searched the Chandra archive and found that 10 W4BCGs have existing data. All observations were obtained using the ACIS instrument in either FAINT or VFAINT mode. Table A.2 summarizes these data.

\subsubsection{X-ray $c_{S B}$ Parameter}

Typically, a central X-ray surface brightness excess is a good indicator of a cool core.[67] Following this idea, Santos et al. 2008 investigated the surface brightness concentration of galaxy clusters in the central region, and propose a parameter, $c_{S B}$, to distinguish between $\mathrm{CC}$ and non-CC clusters.[15]

This parameter is defined as the ratio of the soft X-ray flux within $40 \mathrm{kpc}$ and within $400 \mathrm{kpc}[15]$ :

$$
c_{S B}=\frac{F_{r<40 k p c}}{F_{r<400 k p c}}
$$

These radii are chosen because they result in the largest difference between CC and non-CC clusters. The value for $c_{S B}$ can be divided into three different regimes: non-CC $\left(c_{S B}<0.075\right)$, moderate $\mathrm{CC}\left(0.075<c_{S B}<0.155\right)$, and strong $\mathrm{CC}\left(c_{S B}>\right.$ 0.155).[14, 15] Following Santos et al. 2010, we adopted the 0.5-2.0 keV band for the soft X-ray flux measurement.[68]

Fig. 2.9 shows the values of $c_{S B}$ for all the sources with Chandra observations. As is quite apparent, the value for $c_{S B}$ puts seven out of ten of these objects in the strong CC region, although two might have contamination due to AGN activity. However,

there are three clusters not detected in X-ray at all, which will be discussed later in $\S 2.5 .4$. 


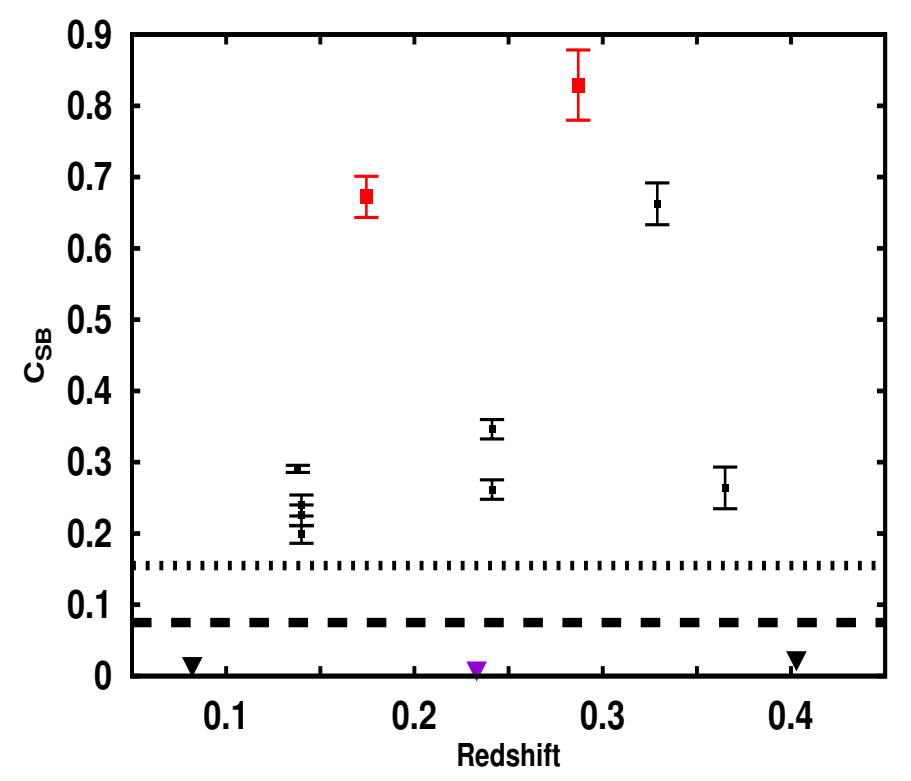

Figure 2.9: A plot of $c_{S B}$ versus redshift of the 10 W4BCGs that have archival Chandra X-ray data. The dashed line at 0.075 separates non-cool-cores from moderate cool cores. The dot-dashed line at 0.155 separates moderate cool-cores from strong cool-cores. Possible AGN hosts are color coded according to identification method: BPT in blue, WISE color in red, and both in purple. The three data points at $\mathrm{z}=0.14$ and the two at $\mathrm{z}=0.24$ are for $c_{S B}$ derived using different observations of the same object. The triangle shows the upper limit of the object that has no X-ray detection.

\subsubsection{X-ray Spectral Fitting}

For these seven CC clusters we further investigate their properties by carrying out Xray spectral fitting. The spectra were fit using XSPEC 12.9 .0 and a cooling flow model mkcflow coupled with a single-temperature mekal model.[69, 70, 71, 72, 73, 74] We follow the same procedure outlined in $§ 3.2$ of Mol16 for the first method to find the spectral mass deposition rate. The minimum temperature for the mkcflow model was frozen to $0.15 \mathrm{keV}$, while the maximum temperature was frozen to $3.0 \mathrm{keV}$. The minimum temperature for the mekal model was set to $4.0 \mathrm{keV}$. The Galactic absorption was frozen to the value based on the radio map of Kalberla et al. 2005 at the position of the BCG.[75] The mkcflow fitting outputs the mass deposition rate $\left(\dot{M}_{d e p}\right)$ in $M_{\odot} / y r$. 
The fitting results are given in Table A.3. Values with no error given represent fits that did not converge (i.e., error was larger than calculated value), and we quote the upper limit based upon the $95 \%$ confidence level. Five out of these seven have mass deposition rates falling short of the SFR estimate from $L_{I R}$. Even for the ones with $\dot{M}_{d e p}>S F R$, it would require a very high efficiency $(\eta>37-86 \%)$ to convert mass into stars so that the observed SFR can be sustained by the cooling flow. Thus, it is unclear whether a possible cooling flow in these CC clusters can be responsible for the observed W4BCG star formation.

This result is in agreement with the recent observations of some BCGs in Mol16, where the mass deposition rate was found to be an order of magnitude lower than the estimated star formation rate. Mol16 provides some possible explanations for this phenomenon, which include an origin of the gas other than the ICM, a delay between cooling and star formation, and, most likely, gas cooling out of the X-ray phase in regions much larger than those measured. However, further investigation is beyond the scope of this research.

\subsubsection{Lack of Cool-Core?}

As shown in the previous section, seven of these W4BCGs are consistent with current theory by residing in cool-core clusters. However, three W4BCG do not have X-ray detection and thus show no sign of being in a cool-core, which could contradict the currently accepted picture. For these objects, the upper limit of the soft X-ray flux within a $40 \mathrm{kpc}$ aperture is no larger than $2 \times 10^{-15} \mathrm{erg} \mathrm{s}^{-1} \mathrm{~cm}^{-2}$ (for comparison,

the detected sources have fluxes on the order of $10^{-13}-10^{-12} \mathrm{erg} \mathrm{s}^{-1} \mathrm{~cm}^{-2}$ ). Here we discuss them briefly: 


\section{$\mathrm{J} 219.69392+06.50142$}

This W4BCG is at $z_{\text {spec }}=0.4029$, which is the most distant one in the X-ray sample. However, its exposure time of only $9.6 \mathrm{ks}$ puts it at the shallow end of observations. Deeper X-ray observations are needed before any conclusions can be reached regarding this particular object.

\section{J355.91977+00.34170}

The lack of X-ray detection at this position may be attributed to the peculiar environment that the cluster resides. This cluster is at $z_{s p e c}=0.261$ (GMBCG gives $\left.z_{p h}=0.23\right)$, and it is merging with a nearby cluster at $z_{\text {spec }}=0.267\left(z_{p h}=0.27\right.$ from GMBCG $)$ that is $5.4^{\prime}$ away. The entire system is usually referred to as $\mathrm{ZwCl} 2341.1+0000$, whose ICM is known to be disturbed and elongated in shape.[76] While there is X-ray emission from the whole structure, the peak lies in between the two clusters and hence is offset from either BCGs. Under this circumstance, it might not be applicable to discuss the existence of a CC cluster.

\section{$\mathrm{J} 125.63314+05.95189$}

While the Chandra exposure at this position is only $8 \mathrm{ks}$, the short integration probably is not the reason for the non-detection because the object is very nearby $\left(z_{\text {spec }}=0.0815^{1}\right)$. In Fig. 2.10, we show that for another W4BCG with a similar exposure time yet higher redshift, there is still a clear X-ray detection. We do note that this particular W4BCG is identified as being in a low-richness or "poor" cluster.

\footnotetext{
${ }^{1}$ This BCG is at $z_{\text {spec }}=0.0815$ based on the SDSS DR7, however GMBCG accidentally does not use this value and keeps quoting $z_{p h}=0.132$ instead. Nevertheless, we verify that it indeed belongs to a cluster at $z=0.08$. It is surrounded by $\sim 12$ red galaxies at $z_{p h}=0.08 \pm 0.02$ that form a clear red sequence, and it is the brightest among all potential members.
} 


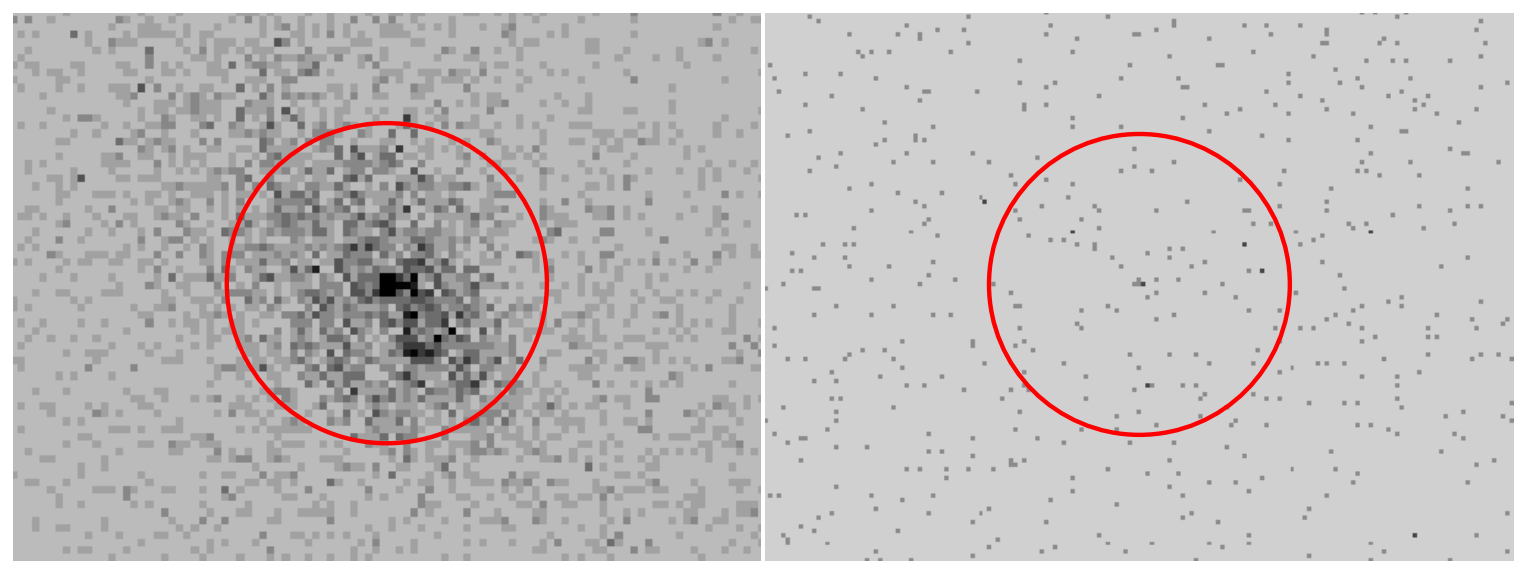

Figure 2.10: The left panel shows J128.72875+55.57253 detected in the Chandra data with an exposure time of $9 \mathrm{ks}$ and $z_{\text {spec }}=0.24118$, while the right panel shows J125.63314+05.95189 not detected in the Chandra data with an exposure time of $8 \mathrm{ks}$ and $z_{\text {spec }}=0.081474$. The circles are centered on the reported SDSS positions and are $40 \mathrm{kpc}$ in size.

Due to the small sample and the aforementioned complications, we conclude that deeper X-ray observations of more W4BCGs are needed in order to put the connection of starforming BCGs and cool-core clusters on a more solid ground.

\subsection{Discussion}

While the common wisdom about BCGs at low redshifts is that they are quiescent galaxies, we have shown that the W4BCGs presented here are exceptions. In most cases, their W4 emissions are due to dust heated by strong star formation. Admittedly, such exceptions will not change the overall picture of BCGs because they are only a minority (W4BCGs accounting only $\sim 0.8 \%$ among the entire GMBCG catalog). However, it is important to understand why such exceptions can happen, because this can be related to the critical question in understanding the evolution of high-mass galaxies, namely, why most high-mass galaxies have their star formation processes quenched early in time.

We first note that the W4BCGs do not seem to have unusual environments. While 
it is widely believed that mergers could induce star formation, the W4BCGs are not predominantly mergers (see $\S 2.4 .2$ ). Second, as compared to the non-W4BCGs, the W4BCGs as a whole have slightly less stellar mass and some of them can have younger ages. However, these differences are not significant and might be the results of the fitting model degeneracy rather than being real.

Third, the cooling-flow interpretation can only explain a small fraction of W4BCGs. Among ten of them that have archival X-ray data, only seven are detected. While these seven X-ray-detected W4BCGs are consistent with being residing in cool-core clusters, five of them have their mass deposition rates (from a cooling flow model) less than their inferred SFRs.

Therefore, our investigations thus far still do not seem to be leading to a universal mechanism that can explain why W4BCGs have high SFRs. Nevertheless, there might be one clue, which is in the redshift distribution of the W4BCGs (see Fig. 2.2). The high-redshift end $(z \gtrsim 0.4)$ of this distribution follows that of the full GMBCG sample, which is not surprising. However, it stays relatively constant at lower redshifts, which is a feature not seen in the redshift distribution of the parent GMBCG sample. While it is still unclear how such a difference can be related to the existence of W4BCGs, it will be worth further study in the future.

\subsection{Conclusion}

In this section, we present our systematic census of BCGs at low redshifts $(z<0.55)$ that are still actively forming stars. We use the SDSS-based GMBCG catalog, which is the largest BCG catalog to date, and identify those that have strong mid-IR emissions by their prominent detections in the W4-band $(22 \mu \mathrm{m})$ in the WISE all-sky survey.

While some of the W4BCGs could be AGN hosts, the majority of them are not. Therefore, their strong W4 emissions should be powered by dust heating from star 
formation. Even for those that are possible AGN hosts, we show that their W4 emissions are still most likely due to star formation. Our W4BCGs have median SFR of $\sim 50 M_{\odot} / y r$, and some have SFR as high as 500-1000 $M_{\odot} / y r$. Clearly, the W4BCGs are quite contrary to what is expected for BCGs at low redshifts, which are believed to be old, passively evolving galaxies (i.e., "red-and-dead"). There have been a number of studies reporting some low-redshift BCGs that still have non-negligible star formation, but their SFRs are lower than what we observe among these W4BCGs and/or have smaller sample sizes. Although such actively star-forming BCGs are only a minority among all BCGs, their very existence could have important implications to the evolution of very high mass galaxies.

Our investigations so far are not able to answer why these BCGs are still actively forming stars at such a late stage. The previous studies of low-redshift BCGs that are still not completely "dead" usually attribute the star formation triggering mechanism to the cooling flows in cool-core clusters. However, for the seven identified to be in cool-core clusters based upon X-ray data, the possible mass deposition rates due to a cooling flow fall significantly short to explain the observed SFRs, and thus the true triggering mechanism still remains a mystery. One possible clue to solve this problem could be that W4BCGs are different from the quiescent majority in their redshift distribution: their number is redshift independent as compared to the whole GMBCG sample. Further study of field galaxies is necessary to shed new light to the understanding of this behavior, which is presented in the following section. 


\section{Chapter 3}

\section{High-Mass Field Galaxies}

\subsection{Introduction}

It is well known that the properties of galaxies, like their morphology, stellar mass, and star formation rate, depends upon its local environment.[77, 78, 79] In the previous chapter, we explored the properties of W4 selected BCGs which reside in cluster environments. Typically, when compared to galaxies in less dense environments, cluster galaxies have lower star formation activity. [80, 81, 82, 83] The W4BCG sample is different in this regard, in that, it has atypically high star formation activity. However, if we compare it to sample of similar high mass, W4 detected galaxies in the field, will its star formation activity be lower in comparison? In order to explore possible environmental effects, a sample of field galaxies is needed for comparison. The following sections outline the procedures taken to acquire such a sample and how it compares to the W4BCG catalog. 


\subsection{Data}

The main data sets used to select star-forming, high mass galaxies are the SDSS based MPA-JHU catalog of spectrum measurements and stellar mass estimates and the WISE all-sky survey data. Specifically, we choose the "un-blurred" version of the WISE data (unWISE) from Lang (2014).[21] A number of sources have far-IR (FIR) data from Herschel, which is used for further analysis. All these data are described in the following sections.

\subsubsection{MPA-JHU Catalog}

We make use of the MPA-JHU ( (Max-Planck Institute for Astrophysics - John Hopkins University) "value-added" DR7 catalog of spectrum measurements based on the SDSS DR7 data.[44, 43, 45] In particular, we use the catalog of stellar mass estimates in order to choose galaxies of high stellar mass $\left(\log \left(\mathrm{M} / M_{\odot}\right) \geq 10.5\right) .[44,84]$ These stellar mass estimates are part of the derived data set of the MPA-JHU catalog. Stellar masses were found using $\chi^{2}$ fitting to models from BC03 based on SDSS DR7 photometry. Additionaly, we will make use of the specific star formation rates (sSFR) based upon the aforementioned stellar masses and the star formation rates based upon emission line measurements included in the derived data catalogs. [43] An even further benefit to using the MPA-JHU catalog is that all of our sources have spectroscopic redshifts.

\subsubsection{SDSS DR12}

Since the MPA-JHU catalog does not include photometry data, we use photometry from SDSS DR12. SDSS DR12 includes all previous data from prior data releases

and photometry is consistent with that of DR7, which the stellar masses are based off of. The main difference between DR7 and DR12 comes from sky coverage, with 
DR12 covering 14,555 square degrees versus 11,663 square degrees for DR7.[85]

\subsection{3 unWISE}

In order to be consistent with the BCG sample, we adopted the unWISE images for visual verification and its forced photometry for quantitative analysis. The forced photometry catalog is based upon SDSS DR10 which has the same sky coverage of DR12 and no changes to photometric data.[85]

\subsection{Selection of High Mass Galaxies with Strong Mid-IR Emission}

As with the W4BCGs, we search for galaxies with ongoing star formation by identiying those that have secure W4 band detection. Here, the sample selection is described in detail.

\subsubsection{Sample Selection}

In order to construct a catalog of high mass sources, a cut is made to the MPA-JHU stellar mass catalog such that all sources have $\log \left(\mathrm{M} / M_{\odot}\right) \geq 10.5$. This subset is then cross-matched with the unWISE forced photometry catalog using a matching radius of $5^{\prime \prime}$. The matched objects are then required to have $\mathrm{S} / \mathrm{N} \geq 10$ in $\mathrm{W} 4$ as reported in the unWISE forced photometry catalog. This results in an initial selection of 31,640 objects.

As before, a visual inspection of the W4 unWISE images is made to ensure that the W4 detection is real. However, due to the large number of sources, visual inspection was done on the subset of galaxies with $\log \left(\mathrm{M} / M_{\odot}\right) \geq 11(6,285$ or $\sim 20 \%)$. The results show that $<10 \%$ of the sample is contaminated by false-detections. We then assume 
that this contamination rate may be extrapolated to the total sample. Therefore, our final sample consists of 31,640 individual galaxies.

\subsection{Data Analysis}

\subsubsection{Possible AGN Hosts}

Following the same procedure as the BCG sample, we want to ensure that the W4 detection is due to star formation and AGN activity. The same diagnostic procedures outlined in $§ 2.4 .1$ are preformed on this sample as well.

\section{WISE Color Diagnostics}

As was done for the W4BCG sample, a color cut of W1-W2 $\geq 0.8$ was chosen to identify possible AGN contamination in the sample.[38] Fig. 3.1 shows a WISE colorcolor diagram of the sample illustrating this cut. In this case, $\sim 5 \%$ of the sample is rejected by this criteria. Individual numbers are presented in Table 3.1.

\section{BPT Diagram}

Since the entire sample was chosen from the MPA-JHU catalog, emission line data is available. Therefore, possible AGN contamination is found using a BPT diagram and the Kauffmann et al. (2003) line. Fig. 3.1 shows the BPT diagram for the entire sample. Unlike the WISE diagnostic, a vast majority $(\sim 56 \%)$ of the sample is rejected due to possible AGN contamination. Further numerical details are presented in Table 3.1. However, there is one caveat here: it is possible that a large portion

of the "AGN" based upon BPT diagram may be low-ionization nuclear emission-line region (LINER) galaxies.[86] The excitation mechanism for LINER galaxies is still an ongoing debate between AGN and star forming regions. 

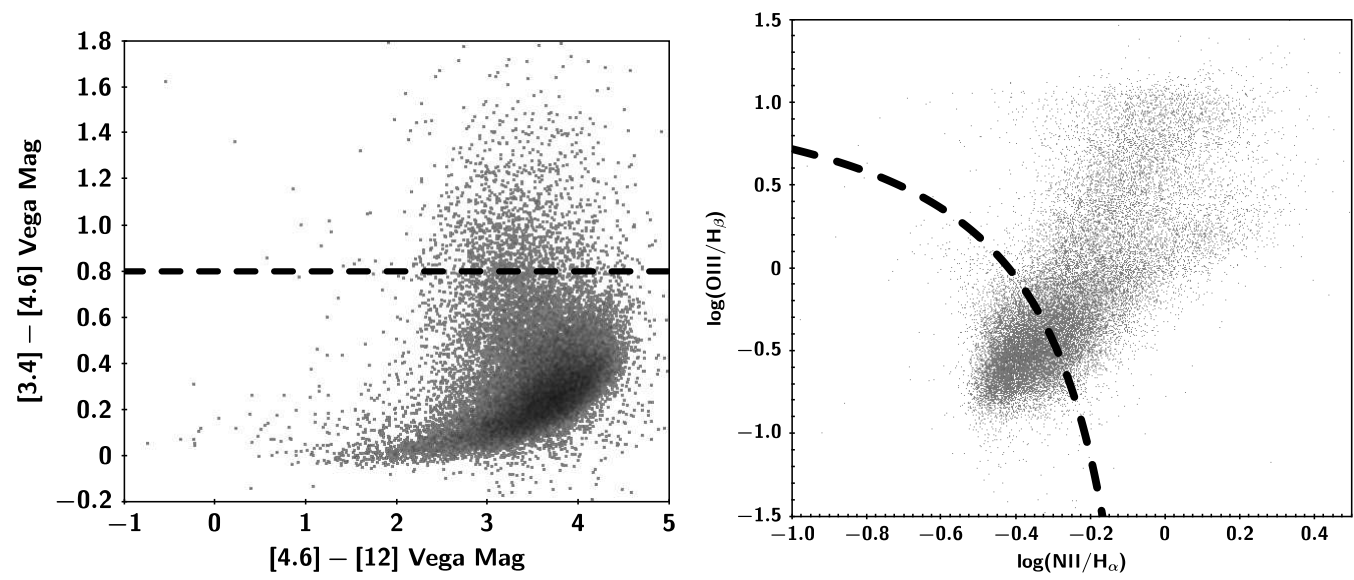

Figure 3.1: Left: The WISE color-color plot for the MPA-JHU sample with the AGN dividing line at $\mathrm{W} 1-\mathrm{W} 2 \geq 0.8$. Right: The BPT diagram for the MPA-JHU sample with the Kauffman+03 line separating star-forming galaxies (below the line) from possible AGN (above the line).

Table 3.1: Possible AGN Hosts Based Upon WISE and BPT Diagnostics

\begin{tabular}{lccc}
\hline \hline & WISE & BPT & Both \\
\hline \# of possible AGN & 1498 & 16492 & 1333
\end{tabular}

\subsubsection{Morphology}

For this sample, we want to separate the sample into early type and late type galaxies (ETG and LTG respectively). Typically, one would expect low-z galaxies with high SFRs to be LTGs. However, this may not always be true, as seen with the W4BCGs. The large sample size makes visual classification tedious and time consuming. In order to remedy this problem, we make use of the SDSS Galaxy Zoo catalog.[87, 88] The Galaxy Zoo project allowed the public to visually classify the morphologies of more than 40 million individual galaxies. The end result of this project is then a catalog which includes the likelihood a galaxy is a certain morphology.

In our case, we are interested in the likelihood that a galaxy is elliptical (ETG) or spiral (LTG). A galaxy was chosen considered to be either elliptical or spiral if the debiased likelihood from the Galaxy Zoo catalog was greater than $50 \%$ in the corresponding morphology category. The morphology breakdown for the total catalog 
and the high mass subset is presented in Table 3.2; keep in mind that some sources were neither identified as elliptical or spiral. It should be noted that the Galaxy Zoo catalog does not contain all of the objects from the MPA-JHU catalog; only 660,645 (71\%) from the total MPA-JHU catalog and 29,566 (93\%) from the high mass subset have Galaxy Zoo data.

\begin{tabular}{rccc} 
Table 3.2: Morphology of MPA-JHU Catalog Based Upon Galaxy Zoo \\
\hline \hline & $\begin{array}{c}\text { MPA-JHU } \\
\text { Catalog }\end{array}$ & $\begin{array}{c}\text { High Mass } \\
\log \left(M^{*} / M_{\odot}\right) \geq 10.5\end{array}$ & $\begin{array}{c}\text { High Mass } \\
\text { W4 Detected }\end{array}$ \\
\hline Total & 660645 & 416262 & 29566 \\
Elliptical & 255897 & 205348 & 4583 \\
Spiral & 159449 & 159449 & 20569
\end{tabular}

\subsubsection{Star Formation Rates}

As previously mentioned, the MPA-JHU catalog also includes star formation rate estimates based upon $H_{\alpha}$ emission line measurements.[43] This would only be an indicator of the un-obscured star formation, and could fail to include dust enshrouded star formation. To remedy this, SED fitting of the IR data is done to estimate $L_{i r}$ and, consequently, the dust enshrouded star formation rate.

\section{SED-fitting}

In order to calculate the $L_{i r}$ and the SFR, we will need to run SED fitting. Again, we use LePhare, to fit the stellar populations and the heated dust components. [51, 52] Input SEDs were constructed using SDSS DR12 photometry along with the unWISE forced photometry. The stellar component is fit to the five SDSS bands and the W1 and W2 bands. The heated dust emission was fit using the templates of Chary \& Elbaz (2001; CE01), and confined to W3 and W4 bands. This is different from the SK07 templates used for the W4BCGs. Since that is the case, for comparison, we 
recalculate the $L_{i r}$ for the W4BCGs using the CE01 templates. A detailed comparison between the $L_{i r}$ calculated by both methods is given in $\S$ B.1. This will allow us to directly compare this sample to the W4BCG sample. The stellar component was fit to the stellar population synthesis models of BC03. We adopted the BC03 models of solar metallicity and the Chabrier IMF, and used a series of exponentially declining star formation histories with $\tau$ ranging from 1 Myr to 20 Gyr. The models were allowed to be reddened by dust following Calzetti's law, with E(B-V) allowed to vary from 0 up to 0.5 mag. Redshifts were fixed to those provided by the MPA-JHU Catalog.

\subsection{Results}
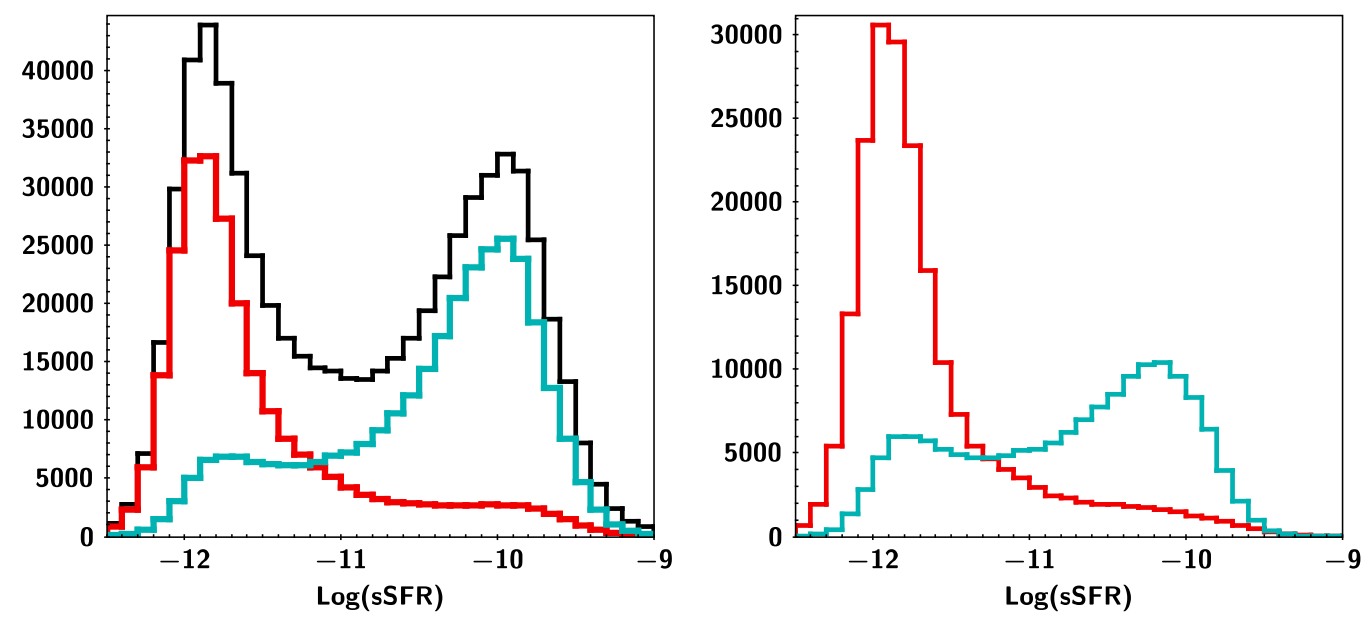

Figure 3.2: Left: The sSFR distribution for the entire sample (black line), the ellipitcal subset (red), and the spiral subset (cyan). Right: Same as on the left except only for the high-mass $\left(\log \left(\mathrm{M} / M_{\odot}\right) \geq 10.5\right)$ galaxies.

As has been discussed by multiple authors (e.g. Brinchmann et al. 2004; Kauffmann et al. 2004; Wetzel et al. 2012; Evans et al. 2018), there exists a bi-modality in the specific star formation rate (sSFR) with a break near sSFR $1_{10}^{-11} \mathrm{yr}^{-1}$ which separates star forming and quiescent galaxies.[43, 89, 90, 91] The bi-modality for the 
entire MPA-JHU sample is presented in Fig. 3.2 Along with that, the sample is broken up into spiral and elliptical galaxies based upon Galaxy Zoo classification. As is expected, the elliptical sample show characteristically lower sSFR's (i.e. massive but quiescent galaxies) and spirals have higher sSFR's meaning they have significant ongoing star formation.

In order to compare to our sample of high mass, W4 detected galaxies, a mass cut is done on the entire MPA-JHU sample. The sSFR for the high mass sample is shown in Fig. 3.2 for the elliptical and spiral subsets. The bi-modality is still present and elliptical galaxies have lower sSFR's than spiral galaxies. From this, we can argue that the bi-modality is independent of mass and exhibits the typical characteristics we expect between elliptical and spiral galaxies.[90]

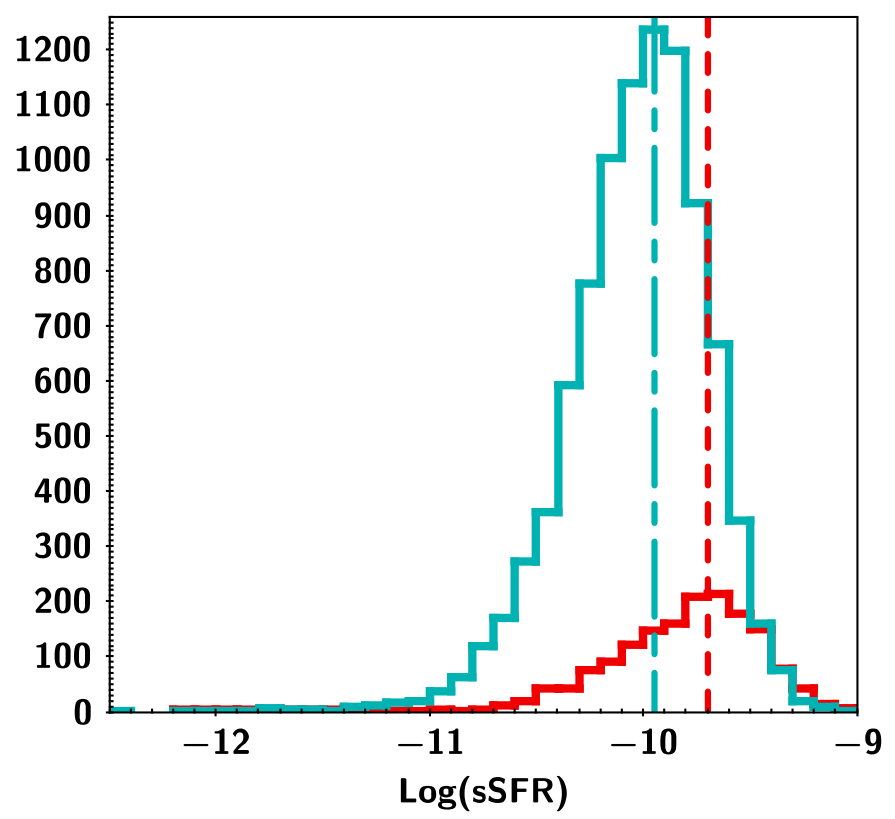

Figure 3.3: The sSFR distribution for the W4 detected, high-mass galaxies. Colors are the same as previous figures. The peak of the distributions are emphasized by vertical lines: dot-dashed at $\sim-10$ for spiral and dashed at $\sim-9.75$ for elliptical.

However, the sSFR distribution for the W4 selected sample, excluding possible AGN hosts, broken up by elliptical and spiral morphology shows little or no bimodality (Fig. 3.3). Based upon the dividing line mentioned above, the W4 selected 

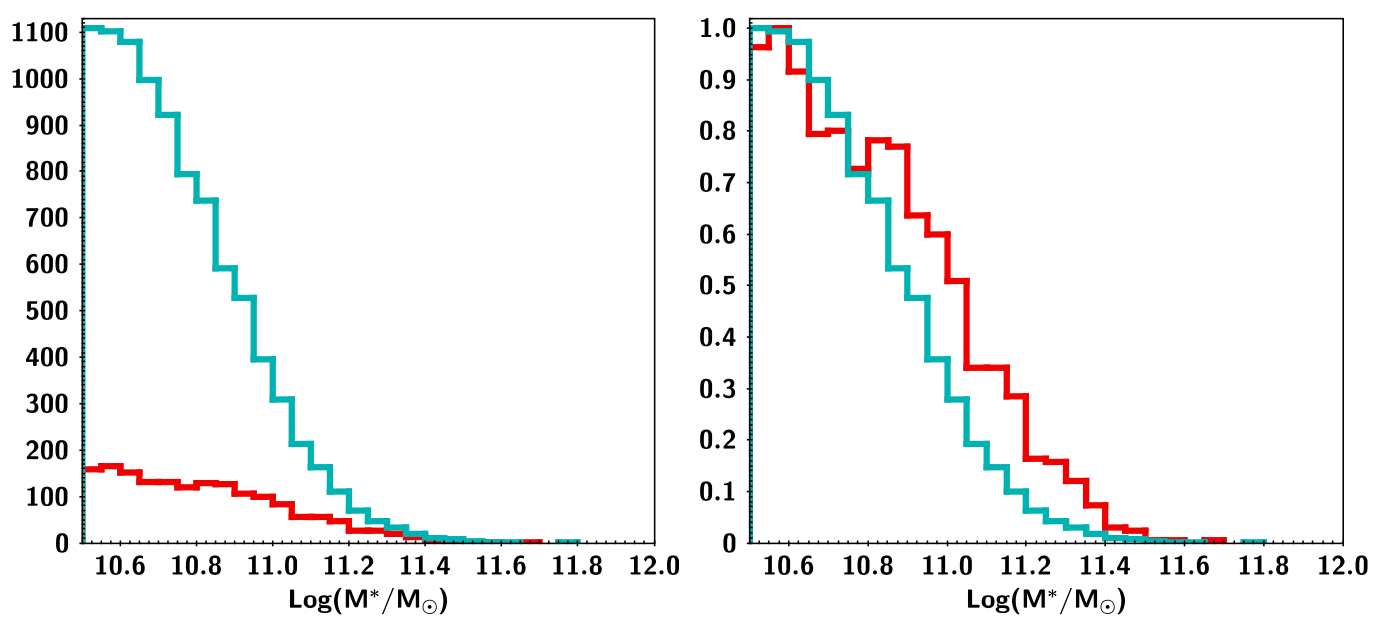

Figure 3.4: Left: The stellar mass distribution for the W4 detected sample separated by elliptical and spiral morphology. Right: A normalized version of the figure on the left for a better comparison.

galaxies appear to be star forming regardless of morphology. Furthermore, the peak of the sSFR distribution for ellipticals is higher $(\log (\mathrm{sSFR}) \sim-9.75)$ than that of spirals $(\log (\mathrm{sSFR}) \sim-10)$. This is quite an unexpected result. The mass distribution (see

Fig. 3.4) for each morphology is comparable; leaving the only cause to be their SFRs. Looking at Fig. 3.5, the peak SFR for ellipticals is double that of spirals ( 12 versus $\left.\sim 6 M_{\odot} y r^{-1}\right)$.

\subsection{Comparison to W4BCGs}

The goal of this research was to create a catalog of high mass, W4 detected field galaxies that could be compared to the W4BCG sample. So that a direct comparison can be made, the field sample is cut to conform to the characteristics of the cluster sample. First, since we are only concerned about W4 detection due to star formation and not AGN heating, both samples only include sources that are not possibly AGN hosts based upon WISE color or BPT emission-line diagnostics. Second, the morphology of BCGs is overwhelmingly elliptical; due to that, only elliptical galaxies from the field sample are considered. Finally, to exclude possible redshift effects, a 


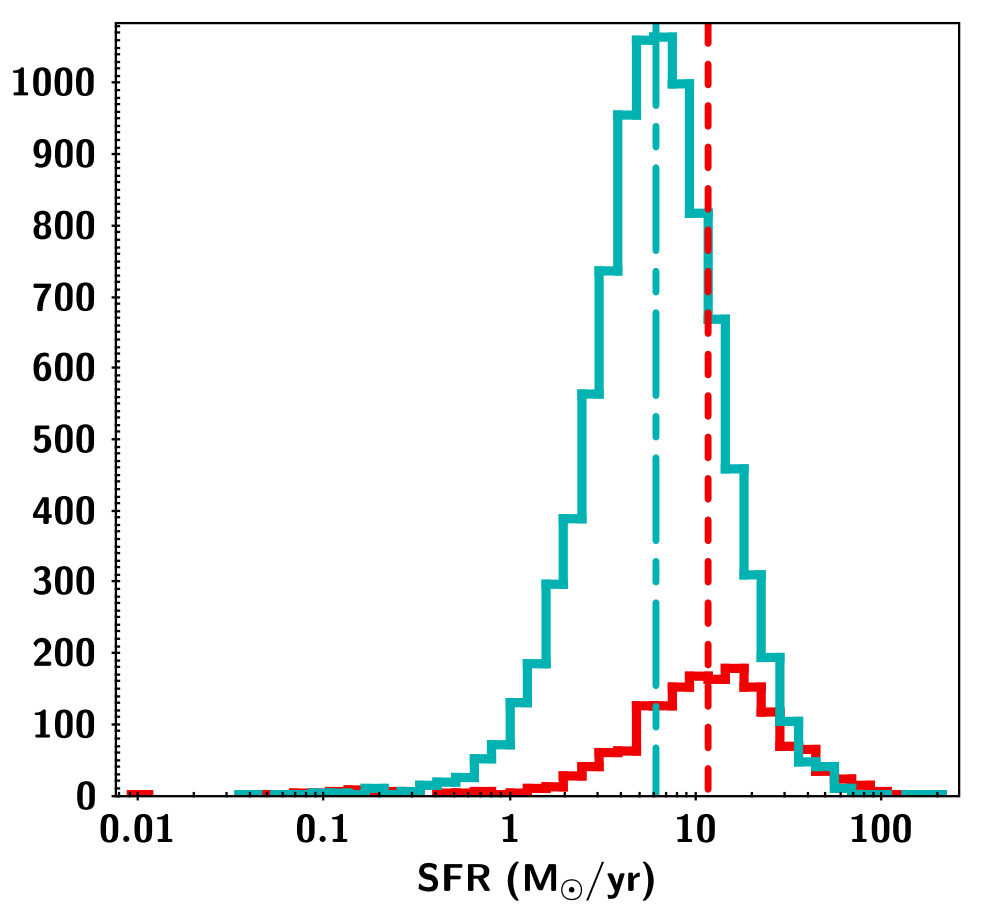

Figure 3.5: The SFR distribution for the W4 detected sample for the elliptical and spiral morphology. Vertical lines are located at the median value of the distribution: dot-dashed at $\sim 6$ for spiral and dashed at $\sim 12$ for elliptical.

redshift range of $0.075<z<0.5$ is chosen. The redshift for each sample is shown in Fig. 3.6. This leaves a sample of 244 W4BCGs and 1,244 high-mass, field galaxies.

The first thing to note is that the median mass of the cluster sample is larger than that of the field sample $\left(\log \left(\mathrm{M} / M_{\odot}\right)=11.2\right.$ and 10.9 respectively; refer to Fig. 3.7). Along with that, the W4BCG sample also has a higher median $L_{i r}$ of $\log \left(L_{i r} / L_{\odot}\right)=$ 11.4 compared to that of $\log \left(L_{i r} / L_{\odot}\right)=11.2$ for the field sample resulting in higher SFRs (see Fig. 3.7). While this may be significant, these differences may not be as apparent when it comes to sSFR. To make this comparison, the $L_{i r}$ is converted to SFR for both samples using Eq. 2.2 and divided by the stellar mass. The results are presented in Fig.Fig. 3.7. As can be seen, both samples fall within similar sSFR ranges, but the median sSFR for the field sample is slightly higher than the W4BCG sample $(\log (\mathrm{sSFR})=-9.7$ and -9.8 respectively $)$.

These results present us with an interesting finding. It appears, regardless of 


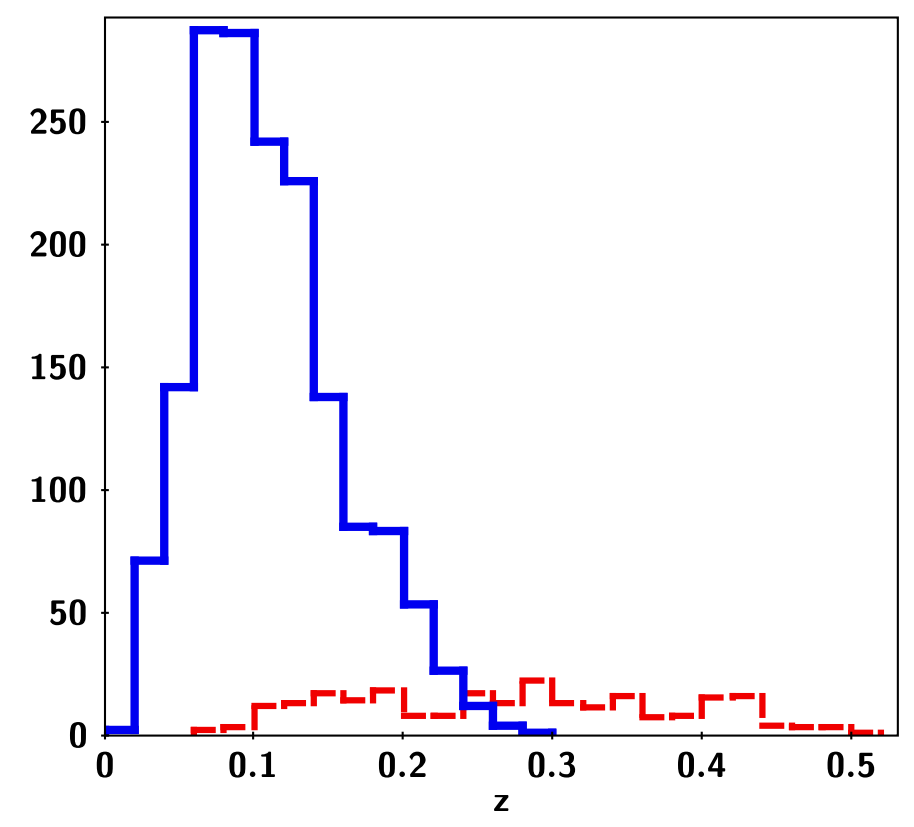

Figure 3.6: The redshift distribution for the MPA-JHU sample (blue solid) and the W4BCG catalog (red dashed).

environment, the W4 detected, elliptical galaxies fall within the star-forming region of the sSFR distribution. Additionally, their sSFRs are only separated by 0.1 dex. Unfortunately, a new problem arises: What is fueling the star formation in field galaxies? For the W4BCGs, a possible explanation is that cooling-flows funnel gas into the galaxy. For field galaxies, no such mechanism is present. Further research, beyond the scope of this thesis, is required in order to identify the mechanism responsible for the ongoing star formation in these massive, elliptical galaxies. A possible clue may be found if one were to analyze their total gas mass (i.e. the fuel supply).

\subsection{Conclusion}

In this section we present a sample of high-mass $\left(\log \left(\mathrm{M} / M_{\odot}\right) \geq 10.5\right)$, field galaxies that are forming stars. The MPA-JHU catalog is used to identify high-mass galaxies and we find those with secure W4-band $(22 \mu \mathrm{m})$ detection in the WISE catalog. Such a selection should identify galaxies with dust obscured star formation. We also break 

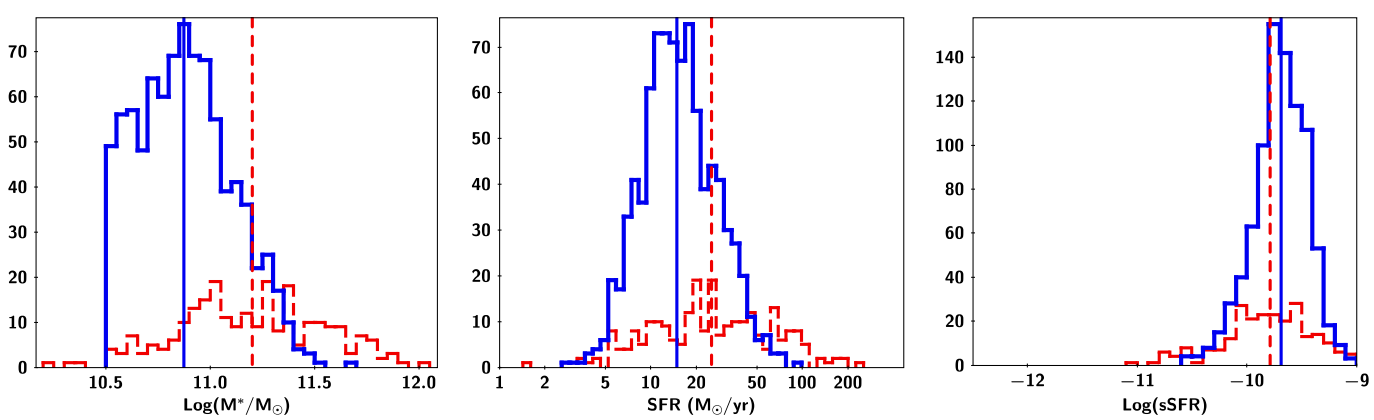

Figure 3.7: Left: The stellar mass distribution for the MPA-JHU sample (blue solid) and the W4BCG catalog (red dashed). Middle: The distribution of the $L_{i r}$ derived SFR for both samples. Right: The sSFR for both samples calculated from $L_{i r}$ based SFR. Vertical lines represent the median values for each sample.

the sample up based upon morphology through the use of Galaxy Zoo.

For the entire MPA-JHU catalog, the bi-modality in sSFR is present, essentially dividing elliptical galaxies from spiral galaxies. However, for the W4 detected galaxies, the bi-modality no longer exists. In fact, W4 detected elliptical galaxies have higher SFRs and sSFRs than spiral galaxies: $\sim 12$ versus $\sim 6 M_{\odot} y r^{-1}$ and $\log ($ sSFR $) \sim-9.75$ compared to $\log (\mathrm{sSFR}) \sim-10$. This is completely reverse of what the bi-modality for the entire MPA-JHU catalog tells us should be occurring.

We also compare this sample of field galaxies to the W4BCGs discussed in the previous section. What we find is that, while the W4BCG sample has a large median stellar mass and SFR, both samples have comparable sSFRs separated by $\sim 1$ dex $(\log (\mathrm{sSFR})=-9.7$ and -9.8 for the field and W4BCGs respectively). It appears that the sSFR is independent of environment for W4 detected, high-mass, elliptical galaxies. While a possible explanation to the SFR for W4BCGs was a cooling-flow, no such mechanism is present for field galaxies. Therefore, further research is needed to identify the process by which these galaxies have ongoing star formation at such a late epoch given their stellar mass. 


\section{Chapter 4}

\section{W3IRDOs}

While visually inspecting the unWISE images for the W4BCG sample, I ran across several instances of sources that would appear in W1, W2, and W4, but were markably missing from the W3 images. An example of such an object is shown in Fig. 4.1. Intrigued by these objects, I set about to systematically find similar objects and investigate possible reasons why they were not detected in W3.

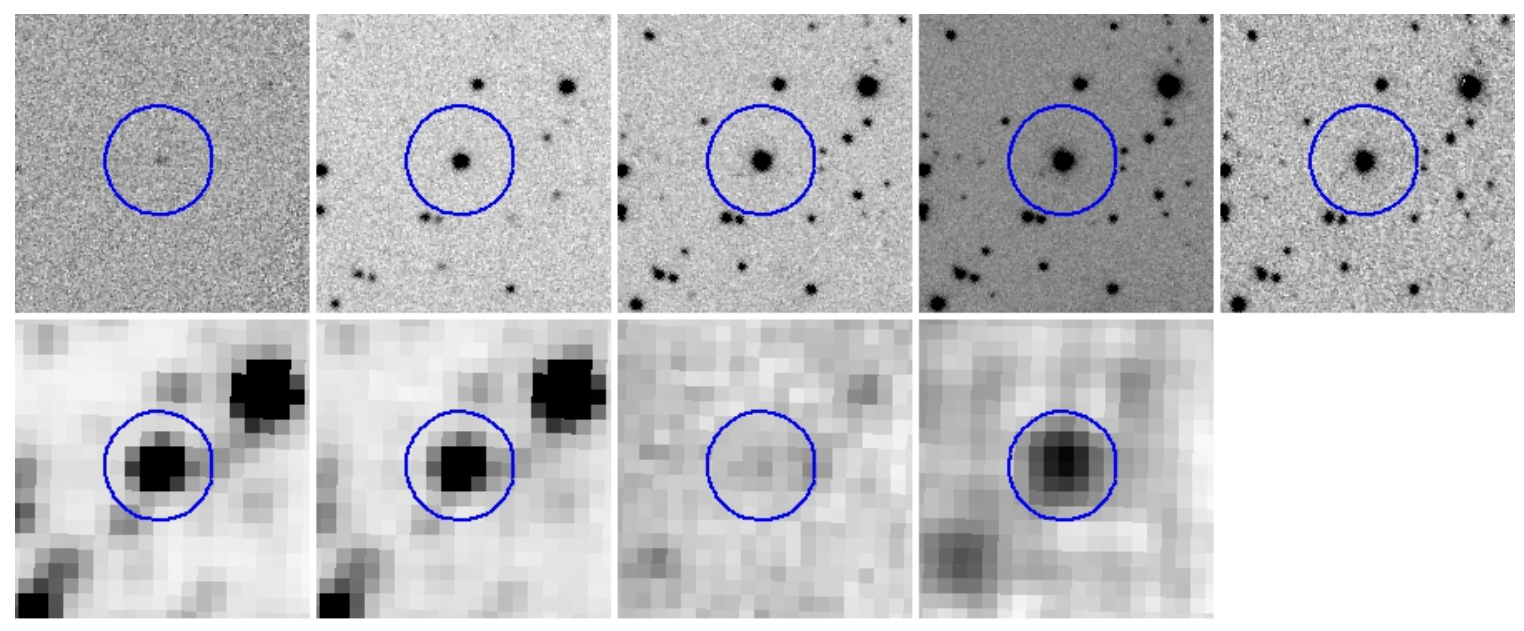

Figure 4.1: An example of a W3 dropout. SDSS ugriz bands are shown on top while WISE bands are shown on bottom. 


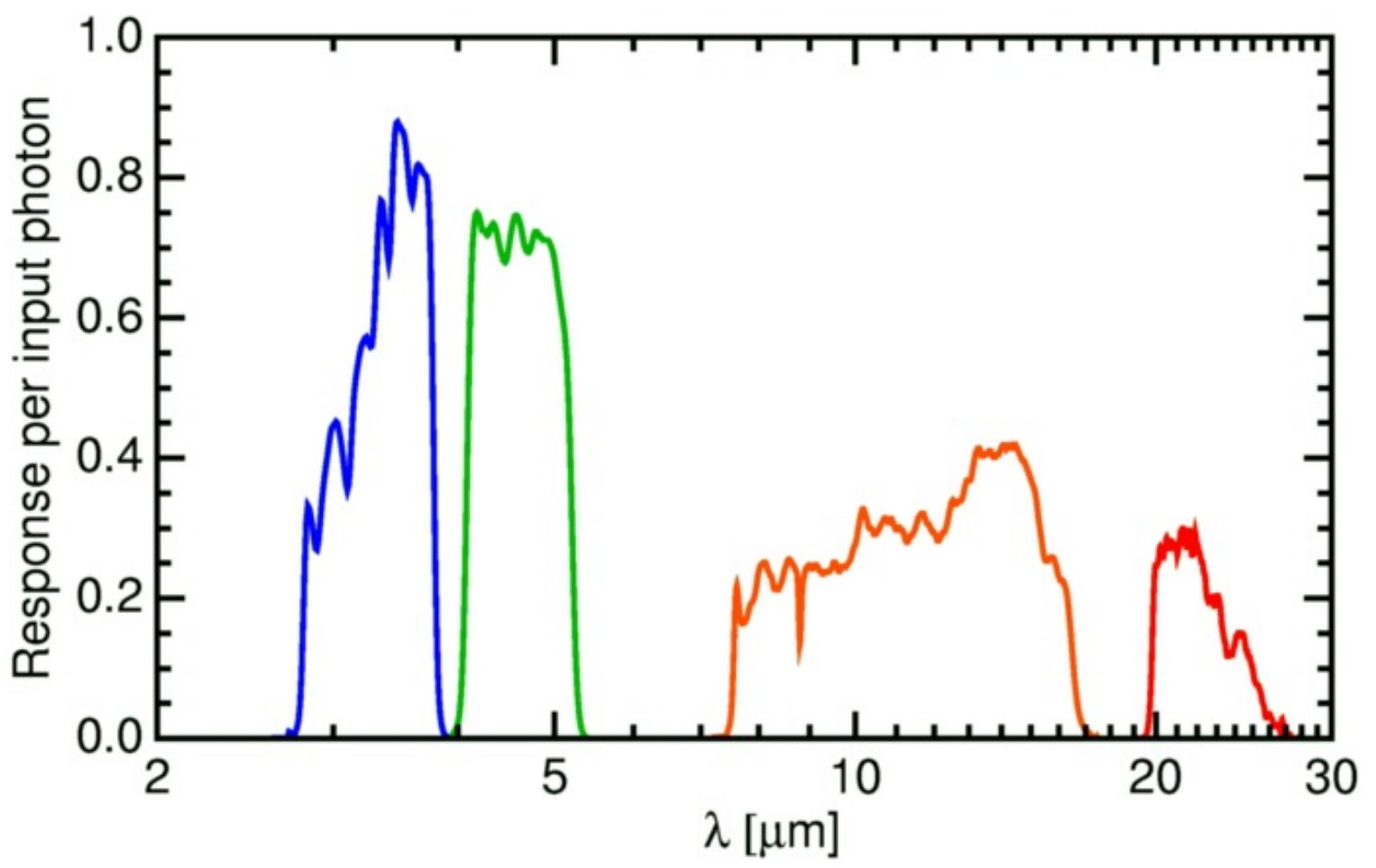

Figure 4.2: The WISE response per photon for W1 (blue), W2 (green), W3 (orange) and W4(red). Courtesy: WISE

\subsection{Why are these sources interesting?}

What makes these sources so interesting comes down to the properties of the WISE passband themselves. As, stated before, the W4 passband has the lowest resolution and sensitivity of all the bands. Therefore, any object detected in W4 should be relatively bright; bright enough to also appear in W3 which has greater sensitivity and also a comparable passband width (See Fig. 4.2). We cannot assume this is an effect due to a high redshift object since it is still clearly detected in W1 and W2. If the peak flux shifted due to redshift, one would assume it would only be detected in the furthest band (W4), which is not the case here. This leaves us with a curious case of an object whose detection or lack thereof is atypical to the norm. Given that these objects are not detected in W3, we identify them as W3 Infrafred Drop Outs (W3IRDOs). 


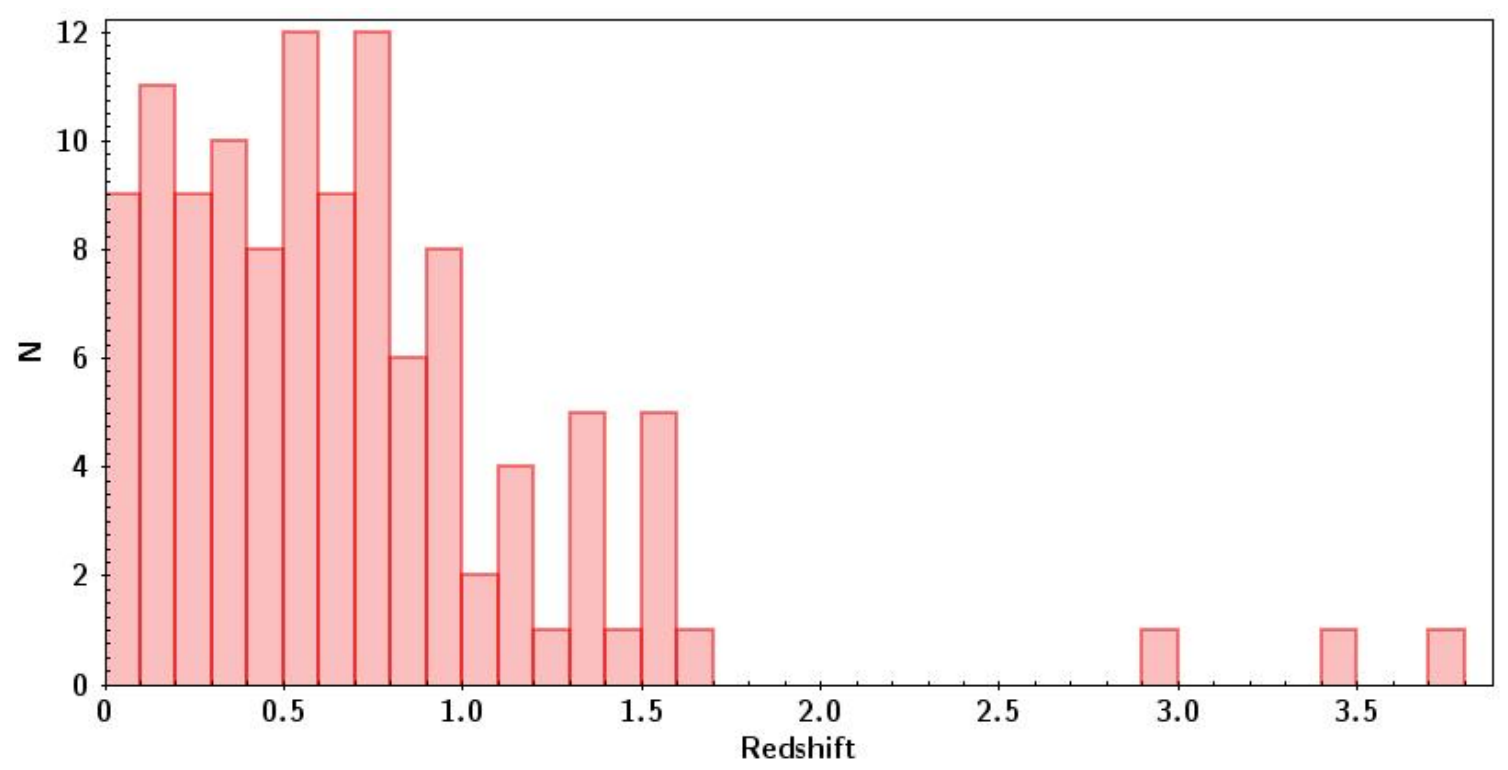

Figure 4.3: The distribution of the photometric redshifts of the W3IRDOs found using the BC03 models.

\subsection{Data}

The data sets used for this investigation were the AllWISE Catalog along with the unWISE images for mid-IR analysis and the SDSS DR12 catalog for optical photometry.

\subsection{Sample Selection}

Using their detection properties, a selection was made using the AllWISE catalog with objects that had the following properties: W1,W2, and W4 SNR $>5$ and W3 $\mathrm{SNR}<2$. This selection should only choose those objects which are detected in W1, W2, and W4 and non-detected in W3. The initial cut results in 282,162 sources. A further cut was made so that only sources that fell within the SDSS footprint and had photometric data were chosen. This was done to ensure that SED could be done to in order to obtain photometric redshift estimates. A total of 7,474 sources satisfy this criteria. 
As done before, we always want to do visual inspection whenever possible to rule out false-positives. Therefore, the unWISE images for all four bands were inspected for each object to ensure a lack of $\mathrm{W} 3$ detection and a secure $\mathrm{W} 4$ detection. After visual inspection, the final sample consists of 116 objects.

\subsection{Data Analysis}

Unlike the previous samples described, we do not have redshifts readily available forthe sources. Due to this, photometric redshifts must be attained with SED fitting. Following a similar procedure to that outlined in $§ 2.4 .3$, the five SDSS bands as well as $\mathrm{W} 1$ and $\mathrm{W} 2$ were fit using the BC03 models by the SED fitting program LePhare. This time, however, redshift was not fixed during the fitting procedure, allowing LePhare to find the most likely redshift based upon the best fit (i.e. photometric redshift). A distribution of the photometric redshifts is shown in Fig. 4.3.

\subsection{Possible Causes}

The source of the W3 dropout likely falls into one of two categories: intrinsic or local. If it is an intrinsic property of the object, the absorption happens at the source and the absorption feature moves into the W3 passband due to its redshift. However, if it is a local effect, the absorption occurs locally (i.e. within the Milky Way) in the W3 passband. The following analysis will try to tackle both cases.

\subsubsection{Intrinsic}

A possible explanation to the W3 drop may be found by examining the spectra of ULIRGs. The strongest features of ULIRG spectra appear in the mid-infrared; most notably, the absorption due to silicate occuring at 9.7 $\mu$ m.[92, 93, 94, 95] This 


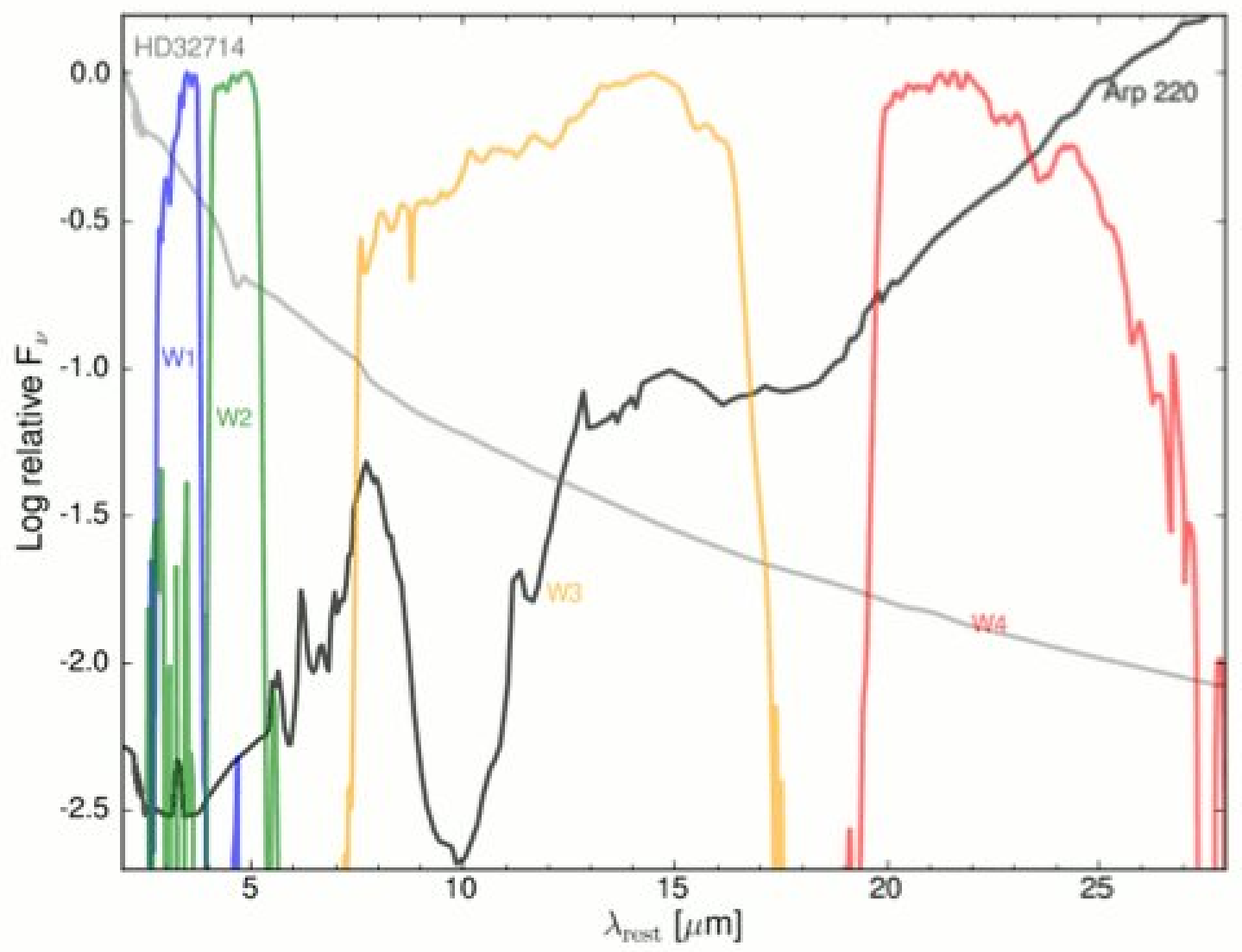

Figure 4.4: The spectra of the closes ULIRG Arp 200 (black solid line) overplotted on the response curves for the WISE passbands. The prominent silicate absorption at $9.7 \mu \mathrm{m}$ falls within the W3 passband. Courtesy: WISE

particular absorption feature has been utilized in previous studies of ULIRGs where it was postulated that sources with strong absorption are heavily obscured by dust.[92, 94, 95] As is illustrated by the spectra of Arp 220, the closest known ULIRG, in Fig. 4.4, this absorption feature falls directly within the W3 passband. For a sanity check, we compare the W3-W4 color of the W3IRDO sample to that of Arp220, which is not a W3IRDO, to see if our objects are redder. The W3-W4 color is 2.2 AB mag and 101 (87\%) W3IRDOs have a W3-W4 color greater than (i.e. redder) than this value, up to $\sim 5.5$. Based on this, it is likely that strong silicate absorption could result in a faint or no W3 detection of a ULIRG.

The intrinsic silicate absorption feature could possibly explain the sample of 


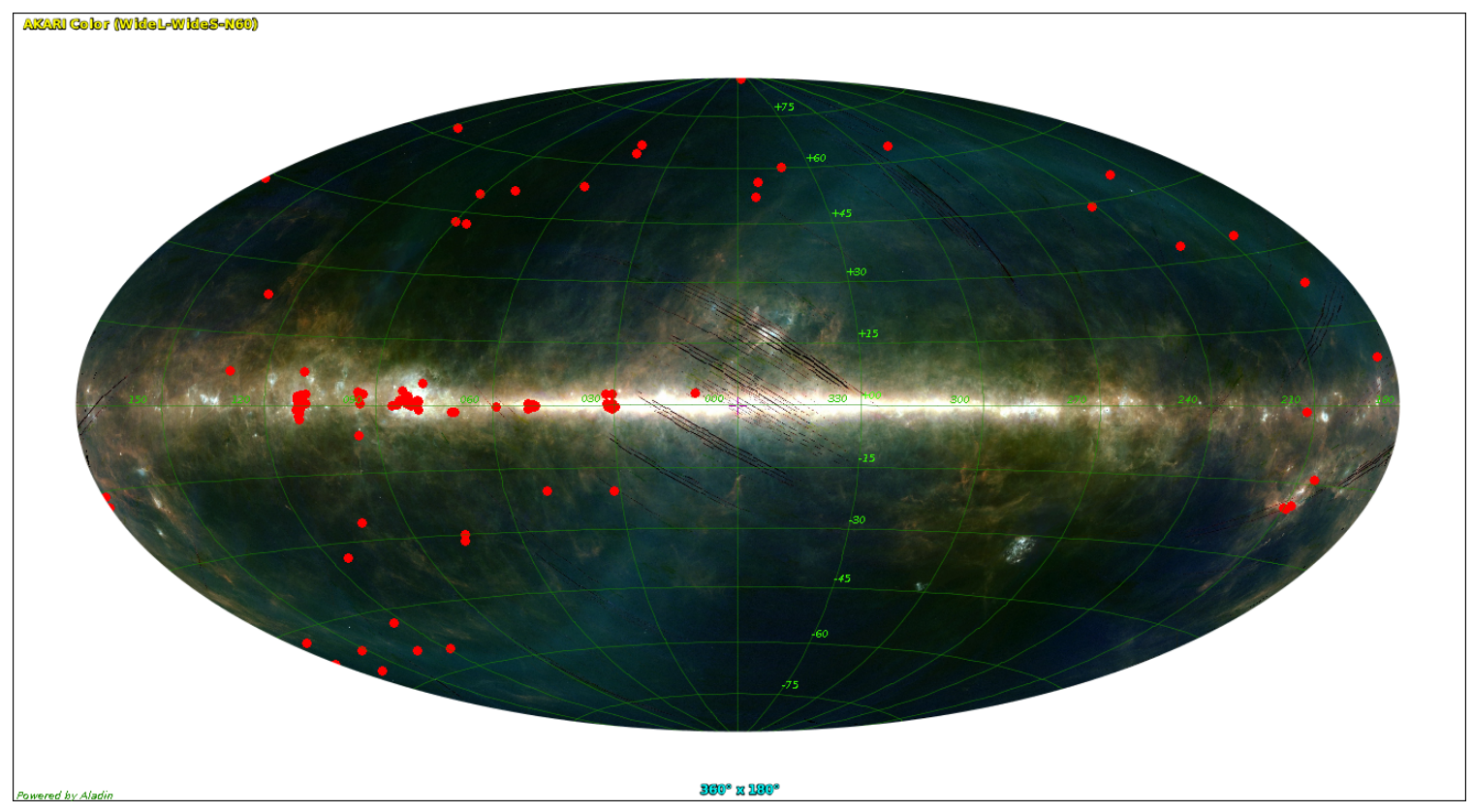

Figure 4.5: The location of the W3IRDOs (red dots) projected onto the sky. A false color image from AKARI is used for illustration purposes.

W3IRDOs, except for one glaring problem. In order for the silicate feature to fall within the W3 passband, the galaxy must have a redshift lower than $\sim 0.7$. As Fig. 4.3 showed, based upon photometric redshift estimates, there are a number of sources that extend beyond this range. To remedy this problem, we must look into a possible local cause.

\subsubsection{Local}

If the absorption within the W3 passband is happening locally, it stands to reason it occurs where there is dense gas and dust. The densest regions of gas/dust within the Milky Way occur along its glactic plane, where the disk lies. By plotting the location of the W3IRDOs using Galactic coordinates, where Galactic latitude $(b)$ equal to zero corresponds to being within the disk, we should be able to see if this hypothesis is correct. As Fig. 4.5 makes clear, a majority of W3IRDOs reside along or near the Galactic plane; a histogram of their Galactic latitudes in Fig. 4.6 illustrates the peak 


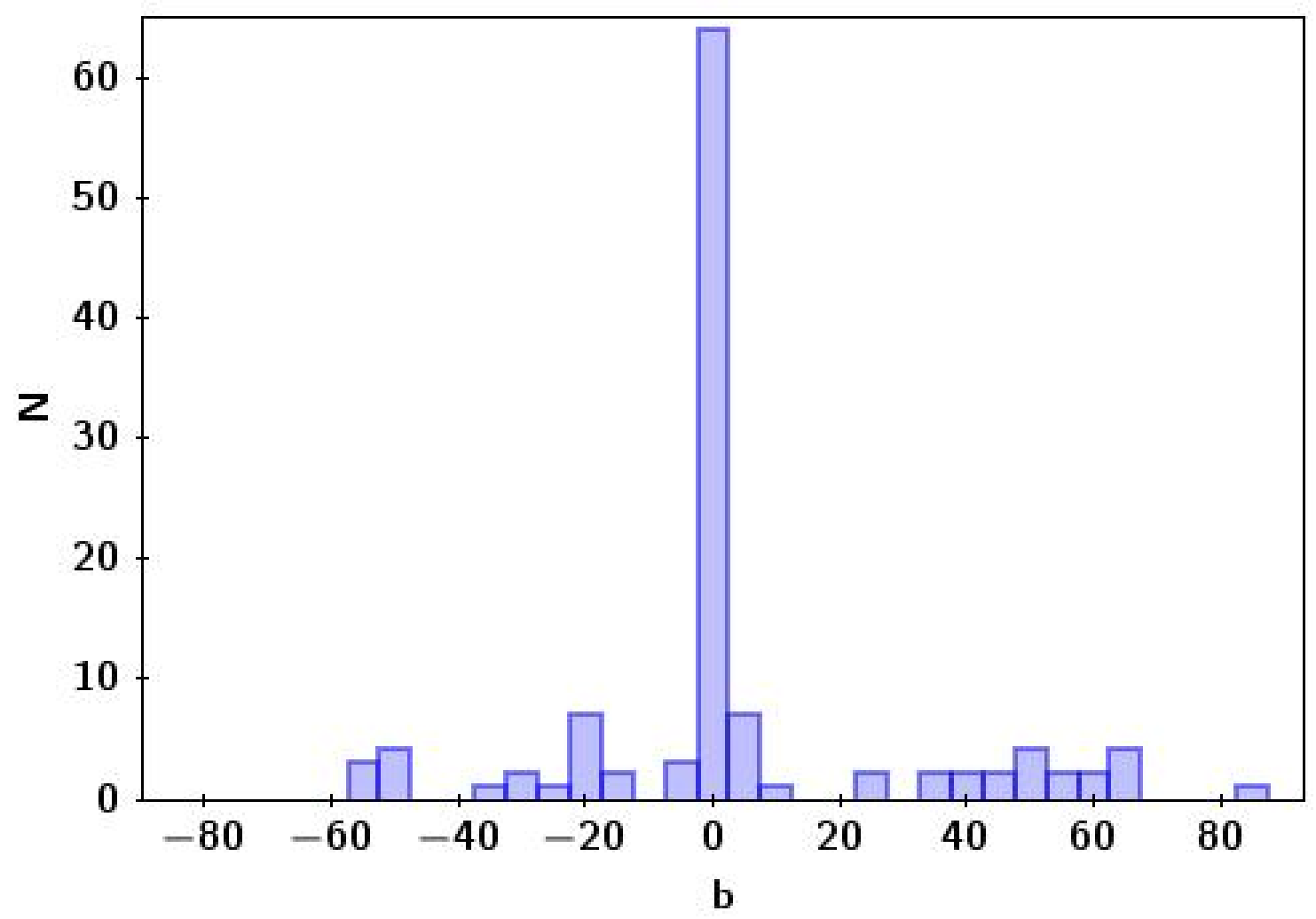

Figure 4.6: The distribution of the Galactic latitude, $b$, for the W3IRDO sample.

density along the Galactic plane ( $65 \%$ have $b \leq 10)$.

While this accounts for a majority of the W3IRDOs, there still exists a large subset that reside at rather high galactic latitudes where the gas/dust density should be significantly lower than that of the disk. At such galactic latitudes, there may exist galactic cirrus clouds. Galactic cirrus clouds are filaments of gas/dust that extend beyond the galactic plane and are named due to their distinct shapes. Due to their composition and temperature, they emit in the far-infarared, as can be seen in the combined image from the Infrared Astronomical Satellite (IRAS) and the Cosmic Background Explorer (COBE) presented in Fig. 4.7. Unfortunately, the resolution of IRAS images $\left(1.5^{\prime}\right.$ per pixel) is not sufficient to reveal possible faint, thin filaments of gas/dust. 


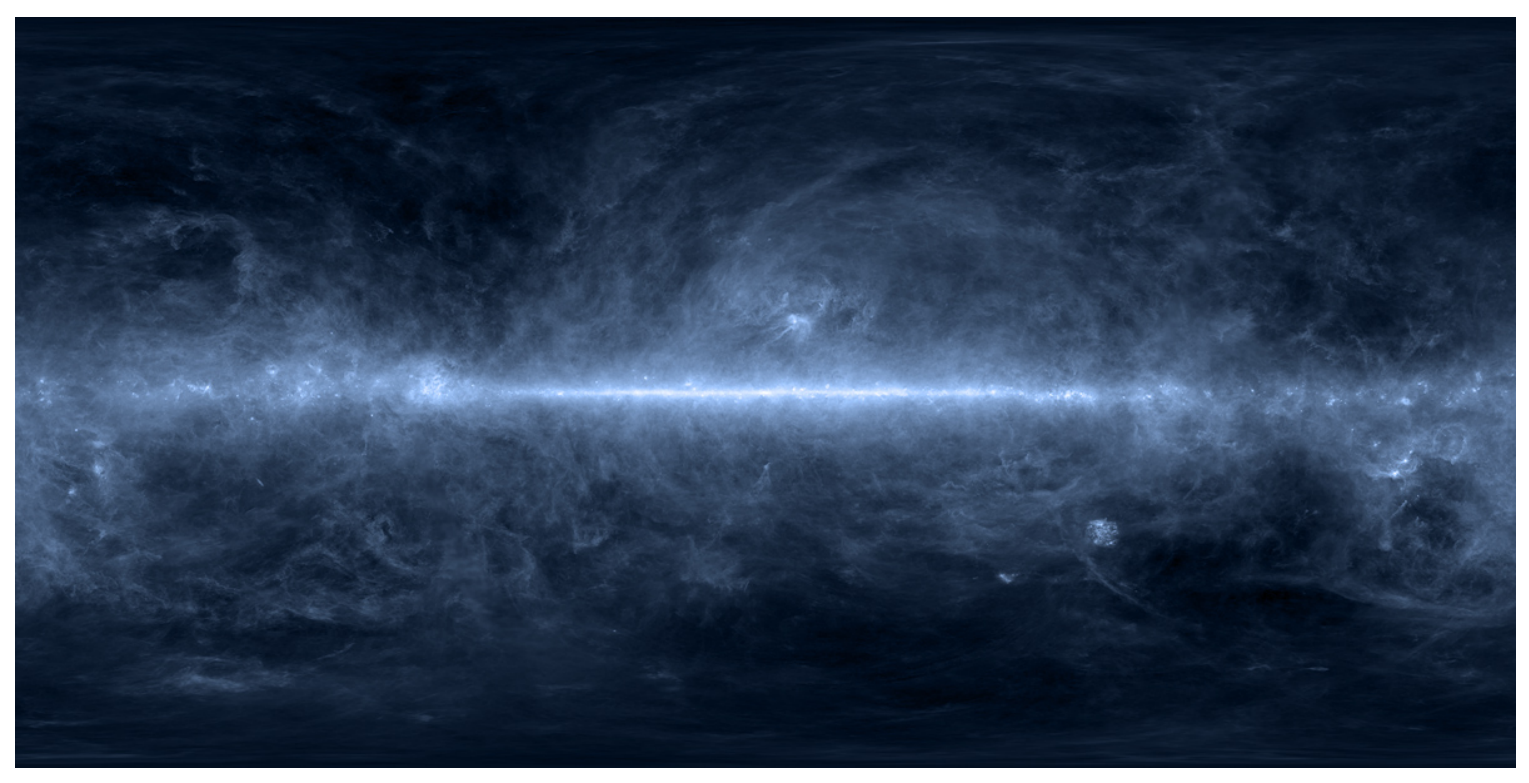

Figure 4.7: A combined IRAS/COBE $100 \mu \mathrm{m}$ map of the sky illustrating the location of gas and dust. The filaments extending beyond the galactic plane are known as galactic cirrus. Courtesy: NASA

\subsection{Conclusions}

A systematic search for W3IRDOs reveals that there are very few of these objects. Two possible explanations as to what is causing the lack of W3 flux still leaves many questions. An intrinsic explanation is possible, but the redshisft and positional distribution of W3IRDOs makes it appear unlikely. However, even a local effect has its drawbacks due to a number of high galactic latitude objects. It may very well be that this sample of W3IRDOs is a mixture of W3 drops due to intrinsic and local effects. Further investigation of W3IRDOs would be needed to verify the exact cause. A first step would be to obtain spectroscopic redshifts instead of depending on photometric redshifts. In addition to that, mid-infrared spectra for these objects would be invaluable to identify what is happening. Therefore, W3IRDOs may be interesting objects for the James Webb Space Telescope (JWST) to view with its Mid-Infrared Instrument (MIRI) which has both a camera and a spectrograph. Until additional data can be acquired, the exact process or processes to the appearance of 
W3IRDOs remains a little out of reach. 


\section{Chapter 5}

\section{Summary and concluding remarks}

In order to investigate the 'downsizing' scenario of galaxy evolution, a sample of highmass, low-z galaxies that still have ongoing star formation was acquired. While these galaxies have red colors in UV, typical of an old stellar population and minimal star formation, mid-IR flux reveals dust-obscured star formation. Using the WISE allsky survey, we choose galaxies with secure $22 \mu \mathrm{m}$ (W4) detection in order to identify galaxies with probable obscured star formation. Both a cluster sample and field sample were chosen in order to see any possible effects the environment plays upon the galaxy properties.

The cluster sample was taken from the SDSS-based GMBCG catalog of BCGs. A full catalog of 389 W4-detected BCGs (W4BCGs) was constructed from the analysis. Through the use of a WISE color-cut and BPT emission line diagnostic, possible AGN hosts were found ( $\sim 20 \%$ of the catalog). Despite this, the W4 emission for the majority of the W4BCGs can be contributed to star formation. Spectral energy distribution fitting reveals that these W4BCGs have a median SFR of $\sim 50 M_{\odot} / \mathrm{yr}$ based upon $L_{i r}$. This is quite contrary to what one would expect according to 'downsizing.' However, BCGs with high amounts of star formation have been known to reside within cool-core clusters, where a çoling-flow is responsible for the enhanced 
SFRs. Analyzing archival Chandra X-ray data supports this assumption; a majority of W4BCGs that have X-ray data do reside within cool-core clusters. While this may be true, the rate at which the cooling-flow deposits mass to the BCG is not enough to account for the observed star formation.

For comparison, a sample of high-mass $\left(\log \left(\mathrm{M} / M_{\odot}\right) \geq 10.5\right)$, field galaxies was chosen from the MPA-JHU emission-line catalog. Since we want to compare this set to the W4BCG catalog, we are mainly interested in looking at elliptical galaxies. The morphology for these galaxies is based upon the Galaxy Zoo catalog. As with the W4BCG sample, possible AGN are rejected using a WISE color-cut and BPT emission-line diagnostic. A bi-modality in sSFR is capable of separating starforming galaxies (spirals) from quiescent galaxies (ellipticals), but the W4 detected galaxies do not exhibit this behavior. The vast majority of the W4 detected subset are star-forming $(\log (\mathrm{sSFR})>-11)$ regardless of morphology. If we compare this sample of elliptical galaxies to the W4BCG catalog, we find that they have similar sSFRs $(\log (\mathrm{sSFR})=-9.7$ and -9.8 for the field and W4BCGs respectively). Therefore, it seems that the sSFR for these galaxies is independent of environment. While the enhanced SFR in W4BCGs could possibly be explained by cooling-flows, there is no such mechanism for field galaxies. Further investigation is needed to find the process/mechanism responsible for the ongoing star formation in these field galaxies.

Lastly, while doing visual inspection of WISE images for the W4BCG catalog, an interesting set of galaxies was discovered based upon their W3 non-detection. Due to this, these objects are named W3 InfraRed DropOuts (W3IRDOs). By doing a thorough search of the WISE all-sky catalog, 116 W3IRDOs were found within the SDSS DR12 footprint. A possible explanation for the non-detection in W3 can be attributed to the silicate absorption feature at $9.7 \mu \mathrm{m}$. If this absorption occurs within the galaxy itself, a redshift constraint of $\mathrm{z}<7$ is necessary. A majority of the photometric redshifts based upon SED fitting fall below this threshold, but there 
exists a small subset of high-z galaxies. For these galaxies, the silicate absorption may be occurring locally, due to the Milky Way. The distribution of the galactic latitude for the W3IRDOs is overwhelmingly along the galactic plane, where the dust and gas density is highest. This lends credence to the hypothesis that W3IRDOs are mainly due to local effects. However, there is still a fraction of W3IRDOs at high galactic latitudes where the dust density is much less than that of the galactic plane. It is possible that thin strands of dense gas and dust known as galactic cirrus can explain these sources, but the sensitivity and resolution of current IR maps is not sufficient to concretely conclude this assumption. Investigation by the James Webb Space Telescope (JWST) could lead to a better understanding of these W3IRDOs, specifically if the absorption occurs intrinsically, locally. Furthermore, there is always the possibility that this sample of W3IRDOs is a mixture of sources due to both types of absorption. 


\section{Appendix A}

\section{Additional W4BCG Data}

\section{A.1 W4BCGs in redMaPPer}

While we focused on the GMBCG catalog for our search of W4BCGs, the same procedure can be applied to other cluster catalogs. One such catalog is that produced by the redMaPPer algorithm.[96] Like the GMBCG catalog, the redMaPPer catalog was produced by the use of a cluster finding algorithm on SDSS data. However, redMaPPer utilized SDSS DR8 photometric data and different criteria for cluster identification. The redMaPPer catalog consists of 26,111 cluster candidates covering a redshift range of $0.08<z<0.55$, roughly half the size of the GMBCG catalog despite covering a larger area and similar redshift range. To check for possible overlap between the two, we cross-matched the catalogs using a radius of $400^{\prime \prime}$, which corresponds to $\sim 2 \mathrm{Mpc}$ at the median redshift $z=0.35$. There is a possible overlap of 14,386 cluster candidates between the redMaPPer and GMBCG catalog.

Following the same procedure outlined in $\S 2.3$, we searched for BCGs in the redMaPPer catalog that had secure W4 detections. The final sample of W4BCGs in the redMaPPer consists of 16 candidates ( $\sim 0.07 \%$ of the total catalog). We pre- 

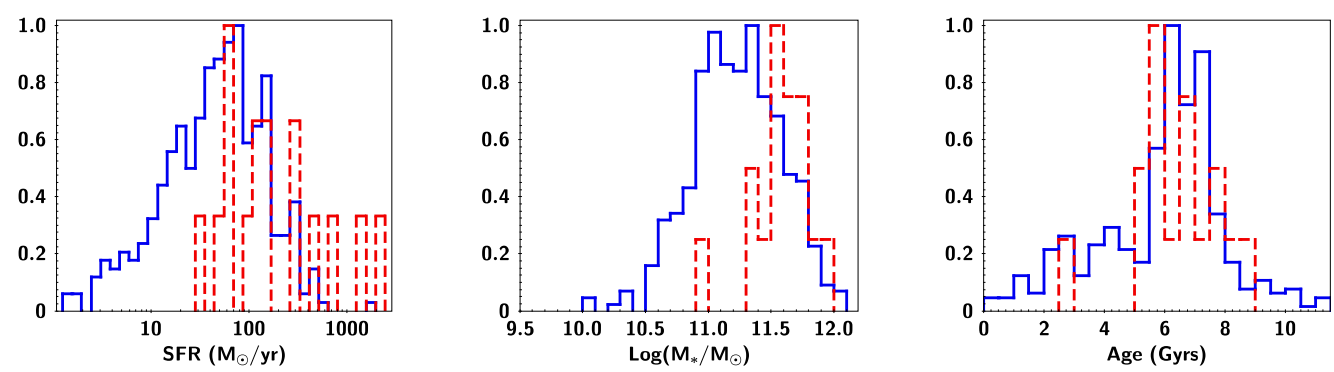

Figure A.1: Comparison of the derived SFR (left), stellar mass (middle), and age (right) for the W4BCGs from the GMBCG catalog (solid blue) and the redMaPPer catalog (red dashed), following the same SED fitting procedure as in $\S 2.4 .3$. The fit to the stellar population is based on $\mathrm{E}(\mathrm{B}-\mathrm{V})$ up to $0.3 \mathrm{mag}$. Each graph is normalized for easy comparison.

formed the same SED analysis for these objects following the procedures in $§ 2.4 .3$. Their $L_{I R}$-based SFR, as well as the stellar mass and the age of their stellar populations, are shown in the histograms in Fig. A.1. For comparison, we also plot the distributions of the W4BCGs from the GMBCG catalog. Despite the small number of sources, it seems that redMaPPer W4BCGs have slightly higher masses and SFRs as compared to the GMBCG sample. However, the fact remains that these W4BCGs exhibit a high amount of star formation based upon their $L_{I R}$.

\section{A.2 W4BCG Tables}




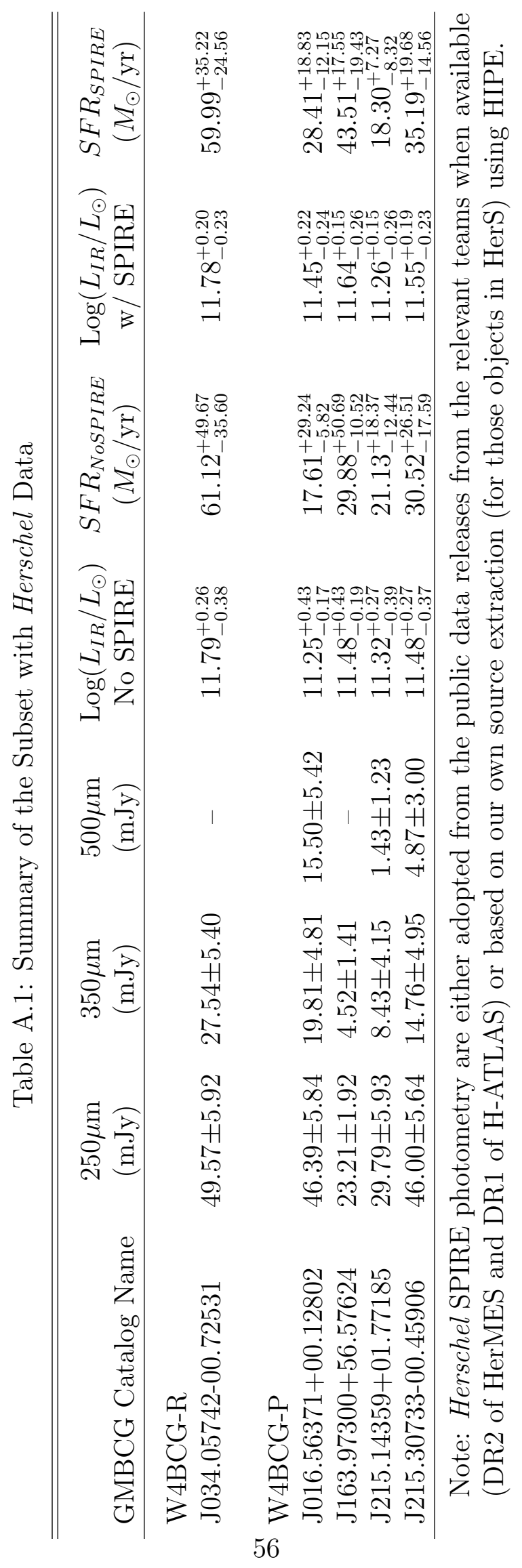




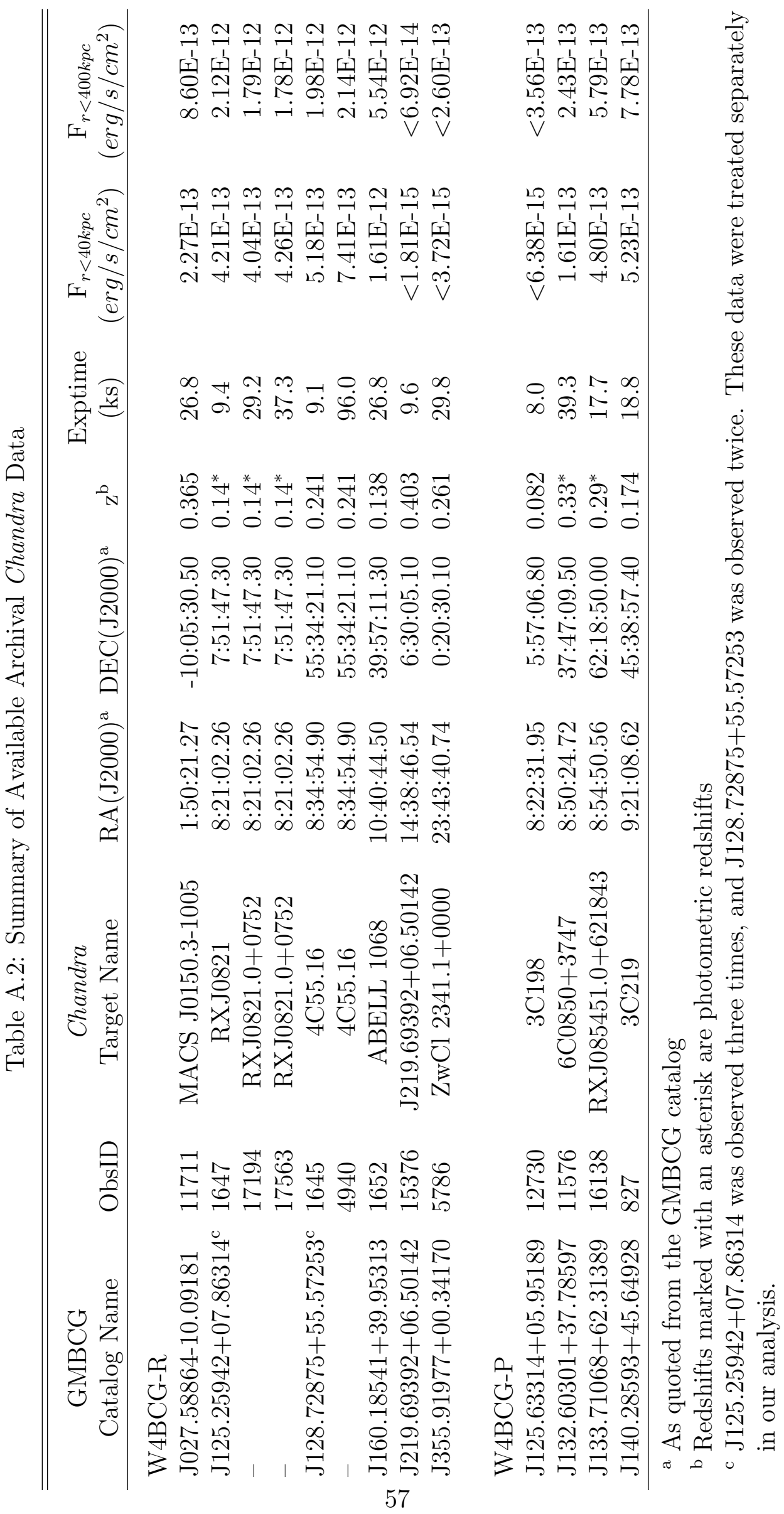




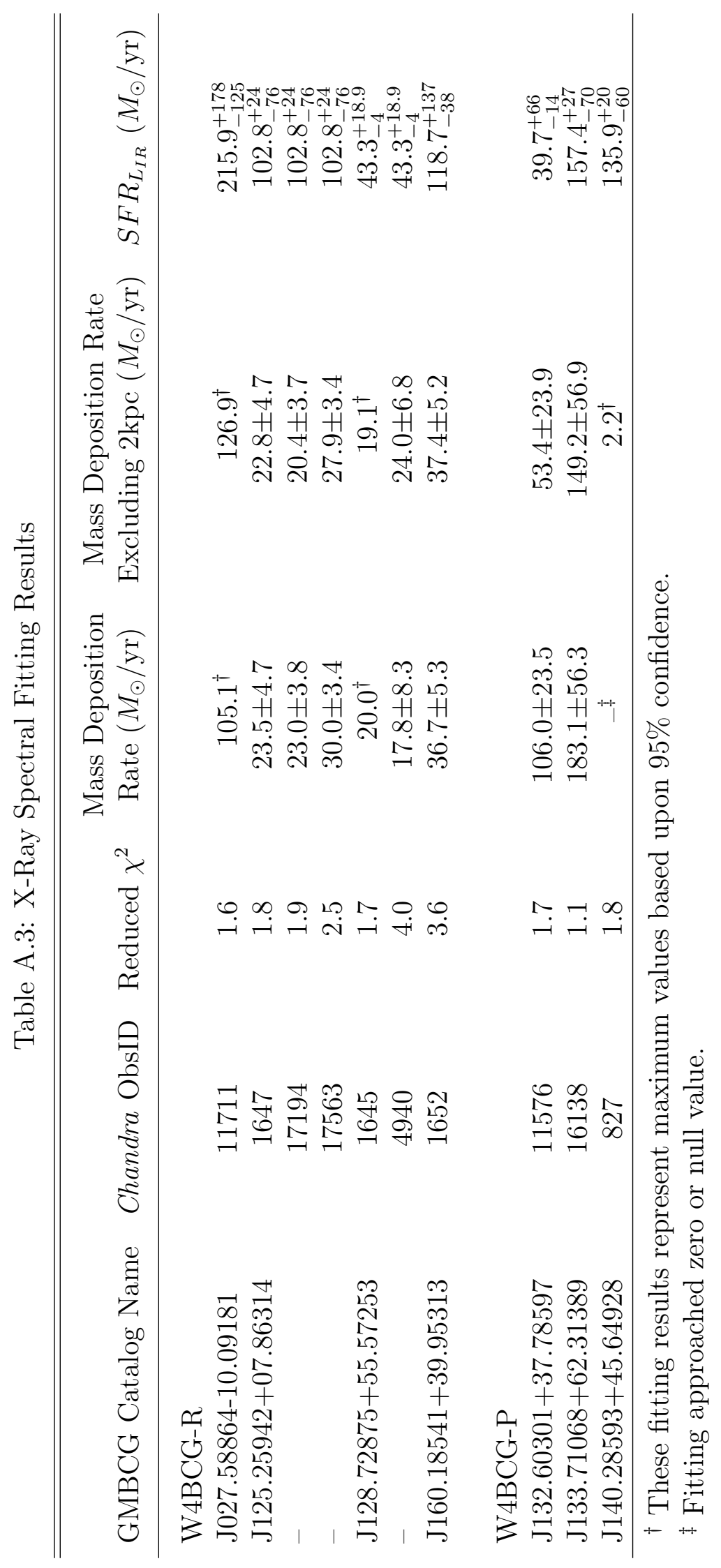




\section{Appendix B}

\section{FIR Templates for MPA-JHU Catalog}

\section{B.1 SK07 vs. CE01 FIR Templates}

While performing SED fitting on the MPA-JHU sample in order to derive $L_{i r}$ estimates, we became aware of an issue when using the SK07 templates. If we plot the $L_{i r}$ versus redshift for the MPA-JHU sample, a discontinuity appears around $\mathrm{z}=0.13$ (see Fig. B.1). This feature appears due to a degeneracy in the SED fitting when these particular FIR templates are used. We re-checked the values for the W4BCG catalog, plotted in Fig. B.1, but there appears to be no discontinuity, possibly due to the low number of sources below $\mathrm{z}=0.13$.

In order to eliminate possible sources of error in the $L_{i r}$ estimate, we choose to use the FIR templates of Chary \& Elbaz (2001; hereafter CE01). Using these templates removes the discontinuity, resulting in a smooth relationship between $L_{i r}$ and redshift (Fig. B.2). For a fair comparison between the MPA-JHU sample and the W4BCG catalog to be made, the $L_{i r}$ values for the W4BCG must be recalculated using the new FIR templates. The difference in $L_{i r}$ values for the W4BCG catalog is shown 

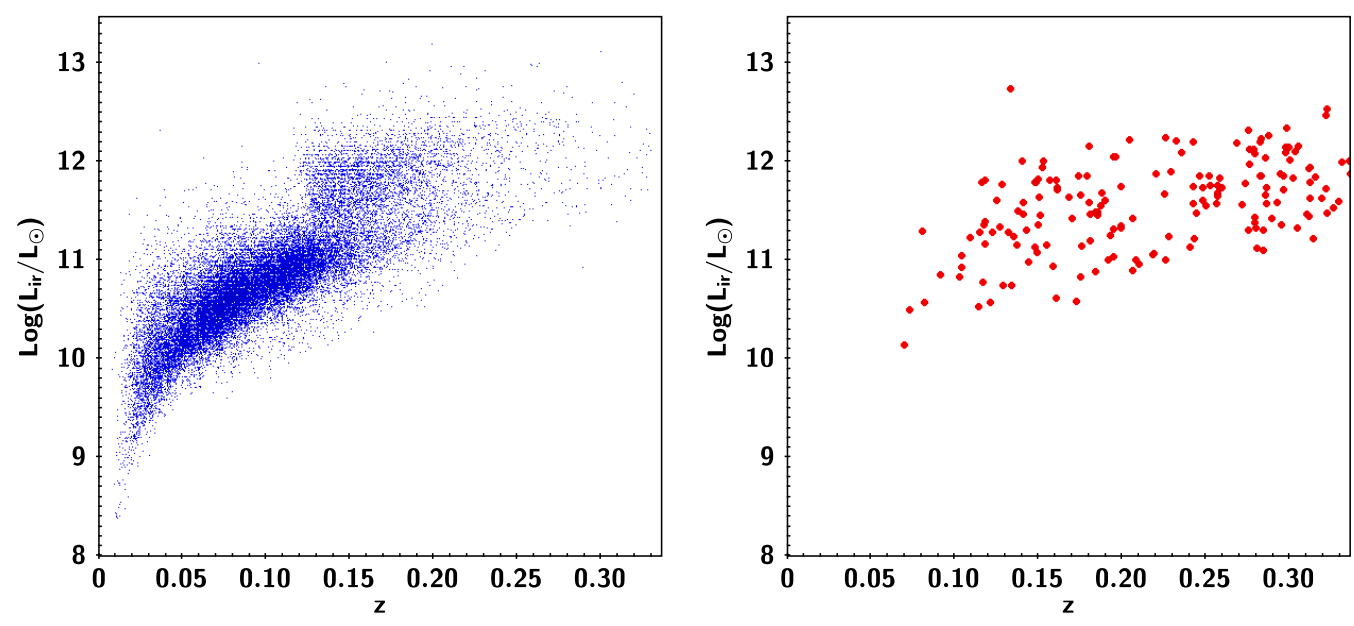

Figure B.1: Left: The $L_{i r}$ estimate based upon the SK07 FIR templates versus redshift for the W4 detected MPA-JHU sample. A discontinuity appears at $\mathrm{z} \sim 0.13$. Right: Same as the figure on the left except for the W4BCG catalog. There is no obvious discontinuity present.

in Fig. B.3. There are two peaks in the distribution: one at 0 and another at 0.5. From this, we can conclude that the $\log \left(L_{i r} / L_{\odot}\right)$ calculated by the SK07 templates either is in agreement with the CE01 templates or higher by 0.5 dex. The difference in $L_{i r}$ will result in higher SFRs for the SK07 templates as compared to the CE01 templates, as shown in Fig. B.3. This will mean that the new SFR estimates for the W4BCG catalog are lower than those reported previously. 


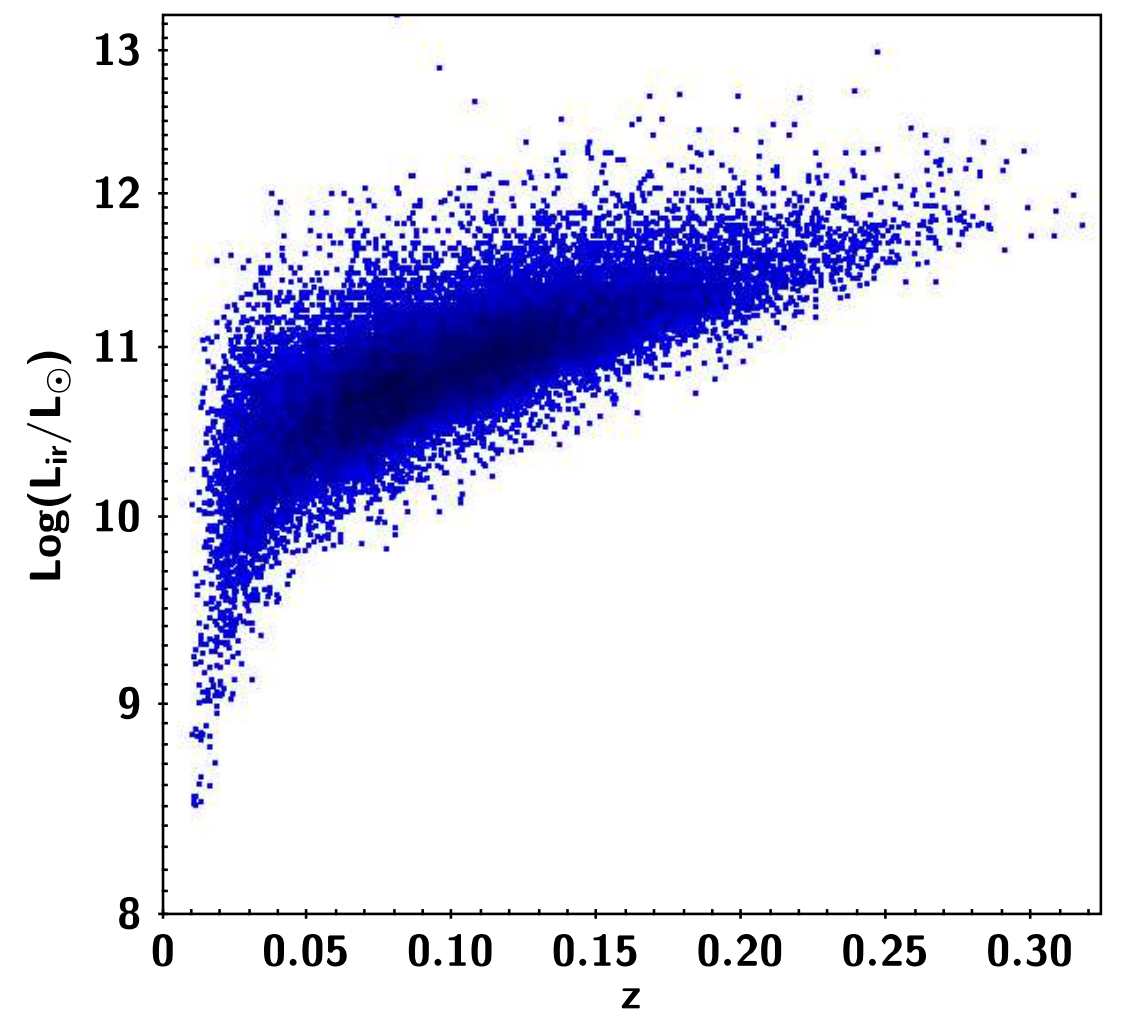

Figure B.2: The $L_{i r}$ estimate based upon the CE01 FIR templates versus redshift for the W4 detected MPA-JHU sample. The discontinuity is no longer present.
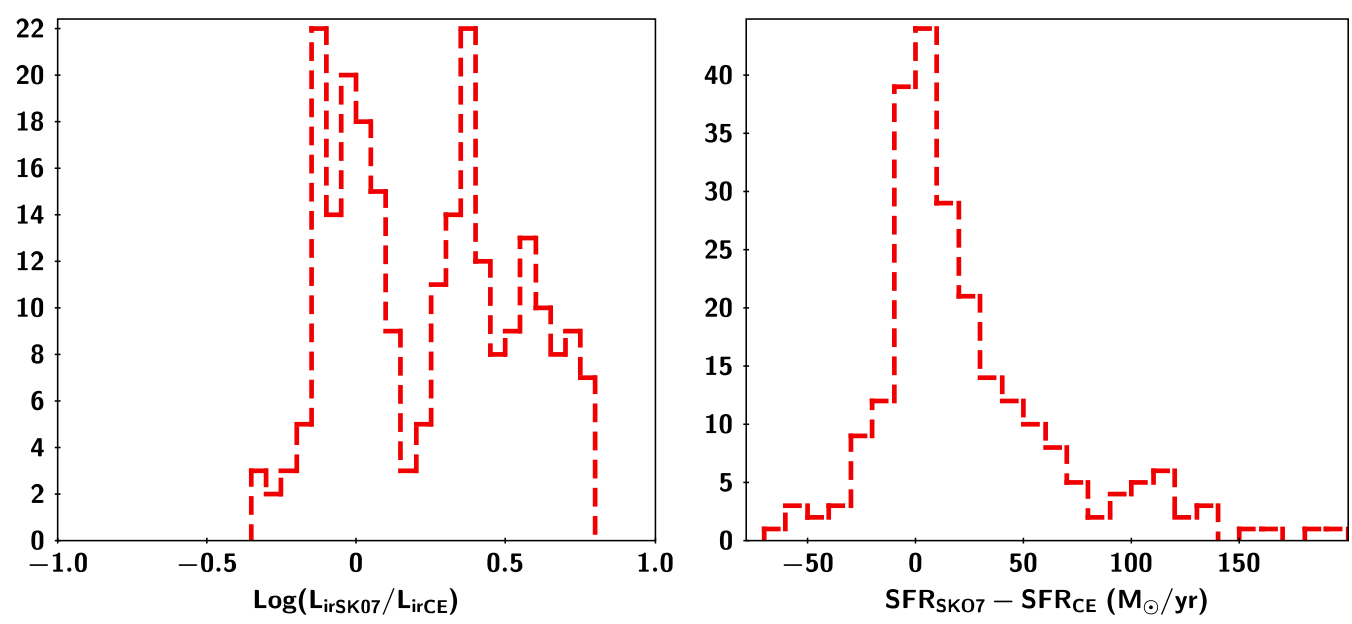

Figure B.3: Left: A distribution of the difference between the $L_{i r}$ calculated by the SK07 templates and the CE01 templates for the W4BCG catalog. Right: The difference between SFR calculated using the $L_{i r}$ values from the figure on the left. 


\section{Bibliography}

[1] L. L. Cowie, Antoinette Songaila, Ester M. Hu, and J. G. Cohen. New Insight on Galaxy Formation and Evolution From Keck Spectroscopy of the Hawaii Deep Fields. AJ, 112(3), 1996.

[2] A. Heavens, B. Panter, R. Jimenez, and J. Dunlop. The star-formation history of the Universe from the stellar populations of nearby galaxies. Nature, 428(6983), 2004 .

[3] S. Juneau, K. Glazebrook, D. Crampton, et al. Cosmic Star Formation History and Its Dependence on Galaxy Stellar Mass. ApJ, 619(2), 2005.

[4] A. E. Bauer, N. Drory, G. J. Hill, and G. Feulner. Specific Star Formation Rates to Redshift 1.5. ApJ, 621(2), 2005.

[5] P. G. Pèrez-Gonzàlez, G. H. Rieke, E. Egami, et al. Spitzer View on the Evolution of Star-forming Galaxies from $\mathrm{z}=0$ to $\mathrm{z} \sim 3$. ApJ, 630(1), 2005.

[6] K. Bundy, R. S. Ellis, C. J. Conselice, et al. The Mass Assembly History of Field Galaxies: Detection of an Evolving Mass Limit for Star-Forming Galaxies. ApJ, 651(1), 2006.

[7] L. Tresse, O. Ilbert, E. Zucca, et al. The cosmic star formation rate evolution from $\mathrm{z}=5$ to $\mathrm{z}=0$ from the VIMOS VLT deep survey. $A \mathscr{E} A, 472(2), 2007$. 
[8] R. C. Kennicutt. Star formation in galaxies along the hubble sequence. ARA\&A, 36(189), 1998.

[9] D. Calzetti, R. C. Kennicutt, C. W. Engelbracht, et al. The Calibration of Mid-Infrared Star Formation Rate Indicators. ApJ, 666(2), 2007.

[10] G. H Rieke, A. Alonso-Herrero, B. J. Weiner, et al. Determining Star Formation Rates for Infrared Galaxies. ApJ, 962(1), 2009.

[11] J. Dubinski. The origin of the brightest cluster galaxies. ApJ, 502(141), 1998.

[12] G. De Lucia, V. Springel, S. D. M. White, D. Croton, and G. Kauffmann. The formation history of elliptical galaxies. MNRAS, 366(2), 2006.

[13] C. O'Dea, S. A. Baum, G. Privon, et al. An Infrared Survey of Brightest Cluster Galaxies. II. Why are Some Brightest Cluster Galaxies Forming Stars? ApJ, 681(2), 2008.

[14] A. Vikhlinin, R. Burenin, W. R. Forman, et al. in Heating versus Cooling in Galaxies and Clusters of Galaxies, ed. H. Böhringer, G. W. Pratt, A. Finoguenov, \& P. Schuecker, 48, 2007.

[15] J. S. Santos, P. Rosati, P. Tozzi, H. Böhringer, S. Ettori, and A. Bignamini. Searching for cool core clusters at high redshift. A\&A, 483(35), 2008.

[16] K. Fogarty, M. Postman, T. Connor, M. Donahue, and J. Moustakas. Star foramtion activity in CLASH brightest cluster galaxies. ApJ, 813(117), 2015.

[17] M. Donahue, T. Connor, K. Fogarty, et al. Ultraviolet morphology and unobscured uv star formation rates of clash brightest cluster galaxies. ApJ, 805(177), 2015.

[18] D. G. York, J. Adelman, J. E. Anderson, Jr., et al. The Sloan Digital Sky Survey: Technical Summary. AJ, 120(1579), 2000. 
[19] E. L. Wright, Peter R. M. Eisenhardt, et al. The Wide-field Infrared Survey Explorer (WISE): Mission Description and Initial On-orbit Performance. AJ, 140(1868), 2010.

[20] J. Hao, T. A. McKay, B. P. Koester, et al. A GMBCG Galaxy Cluster Catalog of 55,424 Rich Clusters from SDSS DR7. ApJS, 191(254), 2010.

[21] Dustin Lang. unWISE: Unblurred coadds of the WISE imaging. AJ, 147(108), 2014.

[22] Dustin Lang, David W. Hogg, and David J. Schlegel. WISE photometry for 400 million SDSS sources. arXiv, 1410.7397, 2014.

[23] M. J. Griffin, A. Abergel, A. Abreu, et al. The Herschel-SPIRE instrument and its in-flight performance. $A \mathscr{E} A, 518(\mathrm{~L} 3), 2010$.

[24] G. L. Pilbratt, J. R. Riedinger, T. Passvogel, et al. Herschel Space Observatory: An ESA facility for far-infrared and submillimetre astronomy. $A \mathscr{E} A, 518(\mathrm{~L} 1)$, 2010.

[25] S. Oliver, J. Bock, B. Altieri, et al. The Herschel Multi-tiered Extragalactic Survey: HerMES. MNRAS, 424(3), 2012.

[26] I. G. Roseboom, S. J. Oliver, M. Kunz, et al. The Herschel Multi-Tiered Extragalactic Survey: source extraction and cross-identifications in confusiondominated SPIRE images. MNRAS, 409(48), 2010.

[27] A. J. Smith, L. Wang, S. J. Oliver, et al. HerMES: point source catalogues from deepHerschel-SPIRE observations. MNRAS, 419(377), 2012.

[28] M. P. Viero, L. Wang, M. Zemcov, et al. HerMES: Cosmic Infrared Background Anisotropies and the Clustering of Dusty Star-Forming Galaxies. ApJ, 772(77), 2013. 
[29] L. Wang, M. Viero, C. Clarke, et al. HerMES: point source catalogues from Herschel-SPIRE observations II. MNRAS, 444(2870), 2014.

[30] M. P. Viero, V. Asboth, I. G. Roseboom, et al. The Herschel Stripe 82 Survey (HerS): Maps and Early Catalog. ApJS, 210(22), 2014.

[31] S. Eales, L. Dunne, A. Cooray, et al. The Herschel ATLAS. PASP, 122(891), 2010.

[32] E. Valiante, M. W. L. Smith, S. Eales, et al. The Herschel-ATLAS data release 1 I. Maps, catalogues and number counts . MNRAS, 462(3), 2016.

[33] S Ott. in ASP Conf. Ser. 434, Astronomical Data Analysis Software and Systems XIX, ed. Y. Mizumoto, K.-I. Morita, and M. Ohishi (San Francisco, CA: ASP), $139,2010$.

[34] A. Fruscione, J. C. McDowell, G. E. Allen, et al. CIAO: Chandra's data analysis system. Proc. SPIE, 6270, 2006.

[35] S. Molendi, P. Tozzi, S. De Grandi, et al. Where does the gas fueling star formation in brightest cluster galaxies originate? A $A\} A, 595(13), 2016$.

[36] T. H. Jarrett, M. Cohen, F. Masci, et al. The SPITZER-WISE survey of the ecliptic poles. ApJ, 735(112), 2011.

[37] S. Mateos, A. Alonso-Herrero, F. J. Carrera, et al. Using the Bright Ultrahard XMMNewton survey to define an IR selection of luminous AGN based on WISE colours. MNRAS, 426(3271), 2012.

[38] D. Stern, R. J. Assef, D. J. Benford, et al. Mid-infrared Selection of Active Galactic Nuclei with the Wide-Field Infrared Survey Explorer. I. Characterizing WISE-selected Active Galactic Nuclei in COSMOS. ApJ, 753(30), 2012. 
[39] R. J. Assef, D. Stern, C. S. Kochanek, et al. Mid-Infrared Selection of Active Galactic Nuclei with the Wide-Field Infrared Survey Explorer. II. Properties of WISE-Selected Active Galactic Nuclei in the NDWFS Boötes Field. ApJ, $772(26), 2013$.

[40] J. A. Baldwin, M. M. Phillips, and R. Terlevich. Classification parameters for the emission-line spectra of extragalactic objects. PASP, 93(5), 1981.

[41] L. J. Kewley, M. A. Dopita, R. S. Sutherland, C. A. Heisler, and J. Trevena. Theoretical modeling of starburst galaxies. ApJ, 556(121), 2001.

[42] G. Kauffmann, T. M. Heckman, C. Tremonti, et al. The host galaxies of active galactic nuclei. MNRAS, 346(1055), 2003.

[43] S. Brinchmann, J.and Charlot, S. D. M. White, et al. The physical properties of star-forming galaxies in the low-redshift universe. MNRAS, 351(1151), 2004.

[44] G. Kauffmann, T. M. Heckman, S. D. M. White, et al. Stellar masses and star formation histories for $10^{5}$ galaxies from the Sloan Digital Sky Survey. MNRAS, 341(33), 2003.

[45] C. A. Tremonti, T. M. Heckman, G. Kauffmann, et al. The Origin of the MassMetallicity Relation: Insights from 53,000 Star-forming Galaxies in the Sloan Digital Sky Survey. ApJ, 613(898), 2004.

[46] G. Chabrier. Galactic stellar and substellar initial mass function. PASP, 115(763), 2003.

[47] Edwin E. Salpeter. The Luminosity Function and Stellar Evolution. ApJ, 121(161), 1955.

[48] R. Chary and D. Elbaz. Interpreting the cosmic infrared background: Constraints on the evolution of the dust-enshrouded star formation rate. ApJ, 556(2), 2001. 
[49] D. Dale and G. Helou. The infrared spectral energy distribution of normal star-forming galaxies: Calibration at far-infrared and submillimeter wavelengths. ApJ, 576(1), 2002.

[50] Siebenmorgen and Krügel. Dust in starburst nuclei and ULIRGs: SED models for observers. $A A, 462(445), 2007$.

[51] S. Arnouts, S. Cristiani, L. Moscardini, et al. Measuring and modelling the redshift evolution of clustering: the hubble deep field north. MNRAS, 310(2), 1999.

[52] O. Ilbert, S. Arnouts, H. J. McCracekn, et al. Accurate photometric redshifts for the CFHT legacy survey calibrated using the VIMOS VLT deep survey. A\& $A$, 457(841), 2006.

[53] G. Bruzual and S. Charlot. Stellar population synthesis at the resolution of 2003. MNRAS, 344(1000), 2003.

[54] D. Calzetti. The dust opacity of starforming galaxies. PASP, 113(1449), 2001.

[55] B. Magnelii, D. Elbaz, R. Chary, et al. The $0.4<\mathrm{z}<1.3$ star formation history of the Universe as viewed in the far-infrared. A\&A, 496(57), 2009.

[56] D. Elbaz, H. S. Hwang, B. Magnelli, et al. Herschel unveils a puzzling uniformity of distant dusty galaxies. $A \mathscr{E} A, 518($ L29), 2010.

[57] D. A. Dale, G. Helou, G. E. Magdis, et al. A two-parameter model for the infrared/submillimeter/radio spectral energy distributions of galaxies and active galactic nuclei. ApJ, 784, 2014.

[58] D. Elbaz, M. Dickinson, H. S. Hwang, et al. GOODS-Herschel: an infrared main sequence for star-forming galaxies. $A \mathscr{B} A, 533(26), 2011$. 
[59] Z. Ma and H. Yan. Co-evolution of Extreme Star Formation and Quasars: Hints from Herschel and the Sloan Digital Sky Survey. ApJ, 811(58), 2015.

[60] S. Molendi and F. Pizzolato. Is the gas in cooling flows multiphase? ApJ, 560(1), 2001.

[61] Y. Chen, T. H. Reiprich, H. Böhringer, Y. Ikebe, and Y. Zhang. Statistics of Xray observables for the cooling-core and non-cooling core galaxy clusters. $A \mathscr{E} A$, 466(3), 2007.

[62] D. S. Hudson, R. Mittal, T. H. Reiprich, et al. What is a cool-core cluster? a detailed analysis of the cores of the X-ray flux-limited HIFLUGCS cluster sample. $A \mathscr{E A}$, 513(A37), 2010.

[63] M. McDonald, B. Stalder, M. Bayliss, et al. Star-forming brightest cluster galaxies at 0.25 ; z ; 1.25: A transitioning fuel supply. ApJ, 817(2), 2016.

[64] M. McDonald, M. Bayliss, B. A. Benson, et al. A massive, cooling-flow-induced starburst in the core of a luminous cluster of galaxies. Nature, 488(349), 2012.

[65] P. Tozzi, F. Gastaldello, S. Molendi, et al. New XMM-Newton observation of the Phoenix cluster: properties of the cool core. A\&\&A, 580(12), 2015.

[66] R. Mittal, M. McDonald, J. T. Whelan, and G. Bruzual. The challenging task of determining star formation rates: the case of a massive stellar burst in the brightest cluster galaxy of Phoenix galaxy cluster . MNRAS, 465, 2017.

[67] A. C. Fabian and P. E. J. Nulsen. Subsonic accretion of cooling gas in clusters of galaxies. MNRAS, 180(479), 1977.

[68] J. S. Santos, P. Tozzi, P. Rosati, and H. Böhringer. The evolution of cool-core clusters. $A \mathscr{G} A, 521(64), 2010$. 
[69] K. A. Arnaud. in Jacoby G. H., Barnes J., eds, ASP Conf. Ser. Vol. 101, Astronomical Data Analysis Software and Systems V. Astron. Soc. Pac., San Francisco, p. 17, 1996.

[70] R. F. Mushotzky and A. E. and Szymkowiak. in NATO ASIC Proc. 229: Cooling Flows in Clusters and Galaxies, ed. A. C. Fabian, 53-62, 1988.

[71] R. Mewe, E. H. B. M. Gronenschild, and G. H. J. van den Ooord. Calculated X-radiation from optically thin plasmas. V. A\&A, 62(197), 1985.

[72] R. Mewe, J. R. Lemen, and G. H. J. van den Ooord. Calculated X-radiation from optically thin plasmas. VI - Improved calculations for continuum emission and approximation formulae for nonrelativistic average Gaunt factors. $A \mathscr{E} A$, 65(511), 1986.

[73] J. Kaastra. An X-Ray Spectral Code for Optically Thin Plasmas (Internal SRONLeiden Report, updated version 2.0), 1992.

[74] D. A. Liedahl, A. L. Osterheld, and W. H. Goldstein. New calculations of Fe L-shell X-ray spectra in high-temperature plasmas. ApJL, 438(L115), 1995.

[75] P. M. W. Kalberla, W. B. Burton, D. Hartmann, et al. The Leiden/Argentine/Bonn (LAB) Survey of Galactic HI Final data release of the combined LDS and IAR surveys with improved stray-radiation corrections. $A \& A$, 440(775), 2005.

[76] R. J. van Weeren, H. J. A. Röttgering, J. Bagchi, et al. Radio observations of ZwCl 2341.1+0000: a double radio relic cluster. A\&A, 506, 2009.

[77] T. Kodama and I. Smail. Testing the hypothesis of the morphological transformation from field spiral to cluster S0. MNRAS, 326(2), 2001. 
[78] G. P. Smith, T. Treu, R. S. Ellis, S. M. Moran, and A. Dressler. Evolution since $\mathrm{z}=1$ of the Morphology-Density Relation for Galaxies. ApJ, 620(1), 2005.

[79] Y. Peng, S. J. Lilly, K. Kovac, et al. Mass and Environment as Drivers of Galaxy Evolution in SDSS and zCOSMOS and the Origin of the Schechter Function. ApJ, 721(1), 2010.

[80] I. Lewis, M. Balogh, R. De Propris, et al. The 2dF Galaxy Redshift Survey: the environmental dependence of galaxy star formation rates near clusters. MNRAS, 334(3), 2002.

[81] P. L. Gomez, R. C. Nichol, C. J. Miller, et al. Galaxy Star Formation as a Function of Environment in the Early Data Release of the Sloan Digital Sky Survey. ApJ, 584(1), 2003.

[82] M. Tanaka, T. Goto, S. Okamura, K. Shimasaku, and J. Brinkmann. The Environmental Dependence of Galaxy Properties in the Local Universe: Dependences on Luminosity, Local Density, and System Richness. AJ, 128(6), 2004.

[83] M. Balogh, V. Eke, C. Miller, et al. Galaxy ecology: groups and low-density environments in the SDSS and 2dFGRS. MNRAS, 348(4), 2004.

[84] S. Salim, R. M. Rich, S. Charlot, et al. UV Star Formation Rates in the Local Universe. ApJS, 173(2), 2007.

[85] S. Alam, F. D. Albareti, C. Allende Prieto, et al. The Eleventh and Twelfth Data Releases of the Sloan Digital Sky Survey: Final Data from SDSS-III. ApJS, 219(1), 2015.

[86] T. M. Heckman. An optical and radio survey of the nuclei of bright galaxies Activity in normal galactic nuclei. $A \mathscr{\mho} A, 87(1), 1980$. 
[87] C. Lintott, K. Schawinski, A. Slosar, et al. Galaxy Zoo: morphologies derived from visual inspection of galaxies from the Sloan Digital Sky Survey. MNRAS, 389(3), 2008.

[88] C. Lintott, K. Schawinski, S. Bamford, et al. Galaxy Zoo 1: data release of morphological classifications for nearly 900000 galaxies. MNRAS, 410(1), 2011.

[89] G. Kauffmann, S. D. M. White, T. M. Heckman, et al. The environmental dependence of the relations between stellar mass, structure, star formation and nuclear activity in galaxiesi. MNRAS, 353(3), 2004.

[90] A. R. Wetzel, J. L. Tinker, and C. Conroy. Galaxy evolution in groups and clusters: star formation rates, red sequence fractions and the persistent bimodality. MNRAS, 424(1), 2012.

[91] F. A. Evans, L. C. Parker, and I. D. Roberts. Red Misfits in the Sloan Digital Sky Survey: Properties of Star-Forming Red Galaxies. MNRAS, 2018.

[92] H. W. W. Sppon, A. G. G. M. Tielens, L. Armus, et al. The Detection of Crystalline Silicates in Ultraluminous Infrared Galaxies. ApJ, 638(2), 2006.

[93] D. Farrah, J. Bernard-Salas, H. W. W Spoon, et al. High-Resolution Mid-Infrared Spectroscopy of Ultraluminous Infrared Galaxies. ApJ, 667(1), 2007.

[94] Lei Hao, D. W. Weedman, H. W. W. Spoon, et al. The Distribution of Silicate Strength in Spitzer Spectra of AGNs and ULIRGs. ApJ, 655(2), 2007.

[95] H. W. W. Spoon, J. A. Marshall, J. R. Houck, et al. Mid-Infrared Galaxy Classification Based on Silicate Obscuration and PAH Equivalent Width. ApJ, 654(1), 2007.

[96] E. S. Rykoff, E. Rozo, M. T. Busha, et al. redmapper. i. algorithm and sdss dr8 catalog. ApJ, 785(2), 2014. 


\section{VITA}

James Runge was born in Des Moines, Iowa. He attended the Illinois Institute of Technology as an undergraduate and received a Bachelor of Science in Physics in 2009. He then enrolled into the Nuclear Engineering program at the University of Missouri in the fall of 2010; for which he received a Master of Science in Nuclear Engineering in December 2011. He was accepted into the Physics and Astronomy Department of the University of Missouri at the beginning of 2012 and is a $\mathrm{PhD}$ candidate. 